\title{
A Soft Systems Approach to Knowledge Worker Productivity: A Purposeful Activity Model for the Individual
}

\author{
Helga Guðrún Óskarsdóttir 1,*(D), Guðmundur Valur Oddsson ${ }^{1}$ (D) Jón Pór Sturluson ${ }^{2}$ (D) \\ and Rögnvaldur Jóhann Sæmundsson ${ }^{1}$ (D) \\ 1 Department of Industrial Engineering, University of Iceland, 101 Reykjavik, Iceland; gvo@hi.is (G.V.O.); \\ rjs@hi.is (R.J.S.) \\ 2 Department of Business Administration, University of Iceland, 101 Reykjavík, Iceland; jonthor@hi.is \\ * Correspondence: hgo2@hi.is; Tel.: +354-6907409
}

Citation: Óskarsdóttir, Helga Guðrún, Guðmundur Valur Oddsson, Jón Pór Sturluson, and Rögnvaldur Jóhann Sæmundsson. 2021. A Soft Systems Approach to Knowledge Worker Productivity: A Purposeful Activity Model for the Individual. Administrative Sciences 11: 110. https://doi.org/10.3390/ admsci11040110

Academic Editor: Isabel-María Garcia-Sanchez

Received: 13 August 2021

Accepted: 17 September 2021

Published: 8 October 2021

Publisher's Note: MDPI stays neutral with regard to jurisdictional claims in published maps and institutional affiliations.

Copyright: (c) 2021 by the authors. Licensee MDPI, Basel, Switzerland. This article is an open access article distributed under the terms and conditions of the Creative Commons Attribution (CC BY) license (https:/ / creativecommons.org/licenses/by/ $4.0 /)$.

\begin{abstract}
This research attempted to find and define holistic systems that affect the productivity of the knowledge worker (KW), using the soft systems methodology (SSM). It is not enough to look at the management and improvement of knowledge worker productivity (KWP) from the viewpoint of the organization. The viewpoint of the individual KW needs to be considered as well. The KW owns the means of production; they carry their knowledge in their heads and take it with them when changing jobs. This paper proposes a conceptual framework that describes the process in which the KW uses resources to execute actions to create tangible or intangible artifacts with the intention of generating value. It was based on interpretations and inferences made from an extensive literature review using the snowballing method. This paper highlights what implications the lessons learned from the conceptual framework have on managing and improving KWP and delves deeper into four key concepts: value in knowledge work, knowledge, personal resources, and competencies.
\end{abstract}

Keywords: knowledge worker; soft systems methodology; productivity; personal resources; value; knowledge; competencies; effectiveness; efficiency; actions

\section{Introduction}

Most jobs today are knowledge based or at least have aspects that are dependent on working with knowledge. The workers in these jobs are called knowledge workers (KWs). KWs have high degrees of expertise, education, or experience and use this to acquire, create, share, or apply knowledge in their jobs (Óskarsdóttir and Oddsson 2017). We are dependent on KWs not only to maintain and keep our economy strong, but also for innovations that will change the world. Knowledge is the key for establishing competitive advantage for many organizations and the key to solving problems that affect us all. KWs are the innovators that will shape the Fourth Industrial Revolution, which is already forcing organizations to reexamine how they do business.

In these modern times, it is becoming increasingly important that we manage the work of KWs to obtain consistent results and improve performance when needed. Organizations and public enterprises need to be able to perceive the productivity of KWs and preferably measure it. Most knowledge is inseparable from the individual, since knowledge is created by interpreting data and information using beliefs, perspective, and experience (Seethamraju 2000). A KW's knowledge cannot easily be assimilated into the organization's process as for manual and routine work. His/her effort needs to be directed to create value for the organization.

Pure manual work and routine work have almost been eradicated by automation. Manual work and routine work that have not yet been automated have been optimized by streamlined processes and systems. There are tried and true frameworks and methods to managing and improving the productivity of manual work and routine work. In knowledge work, however, we are still fumbling around in the dark. Nevertheless, there are 
numerous approaches, frameworks, and methods that are being used to manage and improve knowledge worker productivity (KWP) with varying degrees of success. Their success seems to be context dependent and often fleeting. These frameworks and methods are designed to manage and improve KWP from different viewpoints in the context of the respective challenge at stake.

To give a few examples of the above: The knowledge management approach focuses on codifying knowledge into external knowledge bases with the aim of knowledge sharing, fulfilling information needs, and preserving the organizational knowledge base more easily. Meanwhile, agile management, originally developed for software development, focuses on social interactions, building relationships, and specific planning techniques to collaborate both with customers and colleagues to fulfill requirements. This gives the KWs tools to be more effective. Another approach to managing and improving KWP is nudge management, which was developed at Google. Nudge management draws on insights from behavioral science to design the organizational environment to direct the subconscious behavior of KWs to be more in line with the objectives of the organization (Ebert and Freibichler 2017). Examples of nudges are to provide healthy food in the cafeteria to increase worker wellbeing or design a culture of short meetings by adjusting the default meeting lengths in the software used to book the meetings. These are just a few examples of approaches, frameworks, and methods that organizations are trying to use with differing results to manage and improve their KWP.

All these approaches, frameworks, and methods have something to contribute to optimizing KWP. However, there seems to be lack of a holistic approach to managing and improving KWP. The first step to a holistic approach is to identify the fundamentals of KWP by looking at the problem from a high level of abstraction. There is a vast amount of current research in multiple research fields with a high level of detail that touches on factors that influence KWP. This makes it difficult to obtain a complete holistic view. It is not enough to look at the management and improvement of KWP from the viewpoint of the organization. The viewpoint of the individual KW needs to be considered as well. The KW owns the means of production; they carry their knowledge in their heads and take it with them when changing jobs (Drucker et al. 1997).

There has been a shift of the responsibility of productivity from the organization to the individual, driven by the nature of working with knowledge. Career growth, professional development, and the contribution to the organization have become the worker's own responsibility (Drucker 1999; Letiche and van Hattem 2000). Those who cannot keep up with the demands of the autonomy and accountability resulting from this shift may experience stress and exhaustion (Letiche and van Hattem 2000). Chronic stress can contribute to both physical and mental health problems. Many believe that there is a burnout epidemic because of the fundamental changes in the workplace and the nature of our jobs (Kroft 2020; Maslach and Leiter 1997). Burnout is a state of emotional exhaustion that can lead to anxiety and depression. Burnout makes people feel fatigued and unable to cope with daily tasks, reducing their performance (Maslach and Leiter 1997). Some workers even become unable to work and drop out of the labor force (Salvagioni et al. 2017).

Although the responsibility of productivity has shifted from the organization to the individual KW, the KW is confined and influenced by the systems in his/her environment. The KW's job can be viewed as a process, a process by which the KW uses resources (input), such as knowledge or time, to execute actions to create tangible or intangible artifacts (output) with the intention of generating value (outcome). Evaluating the KW's contribution, in other words the value he/she creates from the effort exerted when executing actions, is quite complex. In this context, the KW's productivity can be viewed as the ratio of value created to effort exerted by the KW while executing the actions that created the value. Both value and effort are subjective. The perception of the KW's productivity is thus influenced by who is perceiving it. What creates value depends on the context, the stakeholders, and their interests and perspectives. It is, therefore, important to have both the efficiency and 
effectiveness of the KW in mind when exploring KWP. Efficiency is about doing things better, while effectiveness is about doing things right.

Value can also be latent. Latent value can, for example, be found in acquiring new knowledge, trying new methods, and other innovative activities. Value is, therefore, not always directly linked to the effort exerted by the $\mathrm{KW}$, making it difficult to measure his/her productivity. Effort is the physical or mental activity performed by the KW. To give an example, under chronic stress, the KW might perceive that he/she needs to exert more effort to generate the same value he/she did before. However, his/her employers might perceive his/her level of effort as the same. This will cause a mismatch in the individual KW's perception of his/her productivity and the organization's perception of his/her productivity.

Óskarsdóttir and Oddsson (2017) found two factions of current research that view the problem of managing and improving KWP from the ideas of their solutions. Both factions assume that effectively working with knowledge is the main factor influencing KWP. One faction believes that the solution is in fulfilling information needs, knowledge sharing, and preventing information overload, making knowledge management the solution. Knowledge management is the process of codifying knowledge into external systems. The other faction believes that knowledge cannot be effectively codified, and therefore, the solution is to invest in the workers themselves through increased motivation, work engagement, and commitment to the organization. Both factions look at the problem of managing and improving KWP from the viewpoint of the organization.

If managing and improving KWP is looked at from the viewpoint of the individual knowledge worker, both the problem description and solutions change. Óskarsdóttir and Oddsson (2017) detected in a literature review of a proxy for industry that individual knowledge workers experience the following challenges as influential to their productivity: too much demand and insufficient resources, choosing what to do and how to do it, self-development, self-awareness, successful relationships, tapping into their full potential, collaboration, the productivity of their thinking, and motivation. Solutions to these challenges have been proposed and used with varying degrees of success. For example: time management, personal resource management, or energy management, key result areas, goals, networking, communication, leadership, motivators, and so on.

Managing and improving KWP has all the hallmarks of a wicked problem. Rittel and Webber (1973) defined a wicked problem as a problem that does not have a fixed definition and has no boundaries. Wicked problems have three things in common (Checkland 2011):

1. They have many viewpoints, which often are competing;

2. These viewpoints are not constant, but change with interpretations of new experiences or knowledge of individuals or groups;

3. The individuals in the situations behave purposefully rather than from intuition or randomly.

In wicked problems, how one understands the problem depends on one's idea for solving it. As Rittel and Webber (1973, p. 162) put it: "One cannot understand the [wicked] problem without knowing about its context, one cannot meaningfully search for information without the orientation of a solution concept, one cannot first understand, then solve". There are no right or wrong solutions, only better or worse from the viewpoint of stakeholders, who all have different interests, values, and worldviews.

It is, therefore, important to view a wicked problem from many viewpoints to identify actions for improvement that are aligned with all viewpoints and do not intensify competing interests (Checkland 2011). The soft systems methodology (SSM) was developed by Checkland (2011) to deal with wicked problems holistically using systems thinking. The SSM consists of four main activities: (1) finding out about a problem situation, (2) formulating purposeful activity models, (3) debating the situation, and (4) taking action for improvement.

This research attempted to find and define holistic systems that affect the productivity of the KW to increase our understanding of KWP, so applicable frameworks and methods can be found to manage, measure, and improve it holistically. We used the SSM to explore 
KWP and formulate a purposeful activity model of the system from the viewpoint of the individual KW. In other words, we executed the second activity in the SSM. Purposeful activity models are tools in the SSM to facilitate structured debates of a situation from different viewpoints (Checkland 2011). The debate is used to find accommodations among conflicting viewpoints. The purposeful activity models are not an accurate representation of the real world, as models built for simulation, but a model of the process of how we explore the world.

Soft systems are described in the form of a transformation process. Purposeful activity models are therefore built by assembling and linking the activities relevant to acquiring the input, the activities to transform it, and the activities to do something with the output (Checkland 2011). Once a purposeful activity model from the viewpoint of the individual has been created, the next step, according to the path set by Óskarsdóttir and Oddsson (2017), is to formulate a purposeful activity model of the system owned by the organization. Only after both of these viewpoints have been explored can the situation be debated and actions for improvement identified. These are the last two activities in the SSM.

A literature review was executed to identify the activities relevant to the process that describes the system from the viewpoint of the individual KW. This is the process in which the KW uses resources to execute actions to create tangible or intangible artifacts with the intention of generating value. The insights gained from this literature review are detailed in the section below. They were used to develop the purposeful activity model and map the identified activities. The purposeful activity model is presented in Section 3 with a discussion about how it was developed. This paper then concludes with a discussion on what implications the insights gained from the purposeful activity model have on KWP.

\section{Literature Review}

A literature review was executed using the snowballing method. The aim was to explore what is value in knowledge work and these three concepts: competencies, knowledge, and personal resources. They were identified as important to the activities of the knowledge worker (KW) when creating value (Óskarsdóttir and Oddsson 2017). This section provides an overview of the results of this literature review.

\subsection{Value in Knowledge Work}

When exploring the question, "What is value in knowledge work?", some conflicting interests become clear. It is not given that what is perceived as value from the viewpoint of the organization is the same as what the KW himself/herself perceives as value. A KW in a modern protean or boundaryless career needs to balance his/her own well-being, career growth, professional development, and contribution to the organization. In some cases, these are aligned, where the same actions contribute to the organization and the KW's needs. Often though, these are in conflict, and the KW must choose actions that contribute to one at the expense of another. Usually in those circumstances, the KW chooses the actions that give him/her the most benefits rather than contributing to his/her organization.

Robertson and Flint-Taylor (2009) stated that it is in the best interest of the organization to ensure that when a KW needs to make decisions or commit high degrees of effort, that the choice, that will maximize his/her well-being, is in alignment with the organization's objectives. KWs find it difficult to make decisions that might damage or deplete their personal resources and would rather commit effort in a task that is psychologically rewarding. Wellbeing at work is derived from positive emotions and an overall sense of purpose that gives direction and meaning to one's actions. The experience of achievement, striving and doing something that is seen as worthwhile, elicits well-being (Robertson and Flint-Taylor 2009).

The organization should, therefore, invest in getting to know their KWs. Then, they can orchestrate their organizational culture, environment, and leadership to maximize value creation that contributes to their objectives and the well-being of their workers (Eriksson et al. 2017). The organization needs to communicate their organizational objec- 
tives and needs to their KWs and motivate them to create value for them. Figure 1 describes this conflict between these viewpoints and how they are communicated.

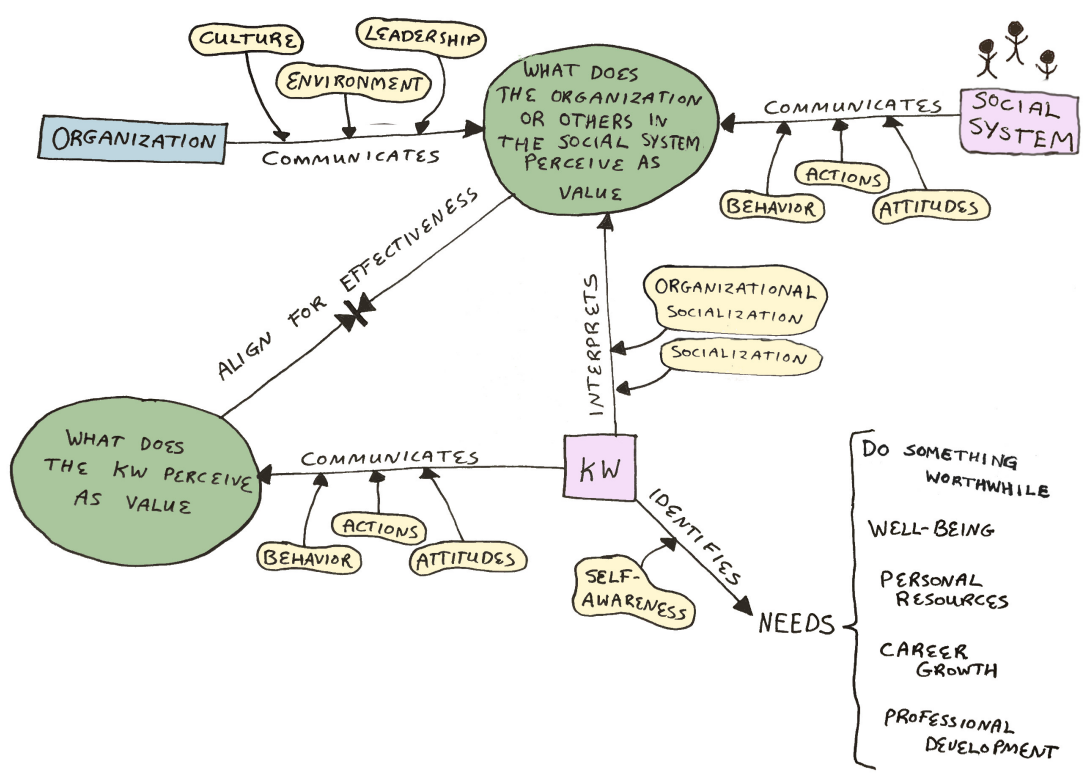

Figure 1. Perceiving value; organization, social system and knowledge worker (KW).

The organization communicates what they perceive as value through their organizational culture, environment, and leadership. The organizational culture conveys how things are done within an organization and what matters (Louis 1980). Leadership manipulates the culture and formulates the work environment (Alimo-Metcalfe et al. 2008). It is vital to learn the culture to develop a scheme for interpreting everyday events within the organization (Louis 1980).

This is usually done through observation and modeling of insiders' behaviors (Jia et al. 2020). By observing successful and competent insiders, the KW obtains information about the appropriateness of imitation and the likelihood of receiving valued outcomes for behaving similarly or expressing similar values (Weiss 1978). This process is called organizational socialization (Cooper-Thomas and Anderson 2006; Louis 1980). Livi et al. (2018) observed that in today's labor market instability, KWs need to keep up with continuous changes within organizations and are often forced to modify their work and social environment. Organizational socialization, therefore, does not only occur in the initial stage of a KW's career anymore, but is a continuous process (Livi et al. 2018).

The KW's social system includes his/her colleagues, personal network, professional network, and community. People in a KW's social system communicate what they perceive as value through their behavior, actions, and attitudes. Creating value for others in your social system can be a source of well-being. For example, helping others can provide positive emotions and contributing to your community can give a sense of purpose. It also builds relationships that can have a restorative effect on the KW's personal resources and gives the $\mathrm{KW}$ access to knowledge he/she might need to create value for the organization. The KW interprets what others in his/her social system perceive as value through socialization. Socialization is a similar process as organizational socialization and allows an individual to integrate successfully into a society, community, or other social groups. Socialization allows a group of people to have a shared cognitive frame and is built on shared experiences, trust, imitation, and observation (Kang et al. 2007; Nonaka 1994; Weiss 1978).

The KW identifies his/her needs using self-awareness, needs that can, for example, be connected to his/her well-being, personal resources, need to do something worthwhile, career growth, or professional development. Self-awareness refers to how conscious an individual is of his/her self-image, own inner state, preferences, personal resources, and 
his/her impact on others (Goleman 1999; Hall 2004). The KW communicates what he/she perceives as value through his/her behavior, actions, and attitudes. The KW takes into account his/her own needs and his/her interpretation of what the organization or others in his/her social system perceive as value when making decisions on what actions to execute, when, and how.

As Óskarsdóttir and Oddsson (2017, p. 12) stated, "the individual knowledge worker cannot fulfill every demand on him, he/she needs to choose what to do, how to do it, and when to do it. The challenge is knowing which tasks to focus on to create value for the organization, the individual knowledge worker or others in his/her social system". The KW is effective when he/she manages to focus on the right tasks to generate his/her intended value, in other words, when what the organization or others in his/her social system perceive as value is in alignment with what the KW perceives as value.

It is also useful for the KW to understand the general mechanisms of economic value creation, to better discern where he/she can contribute to the organization. To create economic value, the outcome of the KW's actions needs to fulfill a need of a stakeholder of the organization in a way that contributes to the organizations objective to realize profits. Economic value can be created by the actions of the $\mathrm{KW}$, but it is only captured when it is exchanged for a profit (Bowman and Ambrosini 2000).

Profits are made when the price paid is higher than the costs of the resources used to create the job, task, product, or service. Therefore, the KW needs to identify actions that contribute to value creation and be able to execute those actions with as few resources as possible. This means that individuals can contribute to value creation by executing actions more efficiently to lower costs (Bowman and Ambrosini 2000). This is also relevant in the public sector even though profit is not the main objective. Public services and enterprises need to do the most general good with the funding that they have. To do that, it is important that their KWs are effective and efficient in creating value for their constituents.

Resources are not only physical, such as materials or equipment, but also intangible, such as the KW's own effort. A KW's effort has a direct monetary cost such as wages, overhead costs, and other benefits the organization pays the KW for his/her time. However, a KW's effort can also have a more indirect cost. For example, a KW that uses too much effort to execute actions can become exhausted, which can lead to a diminished performance, presenteeism, absenteeism, and health problems (Aboagye et al. 2019; Cropanzano et al. 2003).

KWs primarily work for organizations that depend on the ideas and knowledge of their employees for their success (Amar 2004). Innovation is the main competitive advantage of such organizations. Human knowledge is used to innovate what is done and how it is done (Amar 2004), for example creating a fresh approach to an objective or task, improvements to a product, or even a whole new product. It is an investment in possible future value and is therefore inherently risky. Yet, at the same time, successful innovations are the key to an organization's superior profits and growth.

The outcome of innovation is highly uncertain, and the effects of innovation are difficult to measure (Kline and Rosenberg 2009). This creates a challenge when looking at knowledge worker productivity (KWP). It is difficult to discern whether the effort exerted will result in value and, if so, when that value will be realized. This does not mean that a KW that performs innovative actions is unproductive. There needs to be balance in executing actions that create known value and innovating actions that might or might not create value within organizations, to both preserve current profits and grow to realize superior profits.

Innovation can be split into two types-high risk, radical innovation and small, cumulative, evolutionary changes that might reduce costs or enhance a product (Kline and Rosenberg 2009). Both types are important. Control of costs through evolutionary changes is important to remain competitive in the short run, while the movement to radical innovation is often necessary to survival in the long run. An organization needs to nurture an environment that supports cooperation between KWs to leverage their knowledge (Kang et al. 2007). A cooperating environment allows the organization to exploit 
and extend knowledge for competitive advantage and encourage entrepreneurial activity, resulting in radical innovation.

The actions of organizational members are the source of an organizations success, but it is often difficult to contribute specific value to specific organizational members. Their work is interdependent, making the combined result of the KWs' contributions greater than the sum of each individual contribution (Bowman and Ambrosini 2000). It is important to be aware of the level of interdependence. Sometimes, value can be created by helping a colleague, sharing knowledge, and delivering results in a timely manner so that colleagues can use them. These helping behaviors that facilitate organizational productivity by affecting colleagues' performance have been discussed under many concepts such as extra-role performance, organizational citizenship behavior, and contextual performance (Borman and Motowidlo 1997; Cooper-Thomas and Anderson 2006; Podsakoff et al. 1997; Smith et al. 1983; Van Scotter and Motowidlo 1996). This interdependence is explored in the next section about knowledge.

\subsection{Knowledge}

The KW seldom has all the knowledge and information needed to create value. Kang et al. (2007) stated that knowledge is the most distinctive and inimitable resource available to organizations. According to Lee and Yang (2000), "information is data organized into meaningful patterns" and information is transformed into knowledge when an individual understands, interprets, and applies the information in the context of his/her unique personal experiences, lessons learned, judgments, and intuitions.

Polanyi (1966) stated that knowledge has a tacit element and an explicit element, that is "we can know more than we can tell"(p. 4). The explicit element is what we can tell, while the tacit element is what we know that we cannot identify to tell. It is hidden, highly personal, and context dependent (Nonaka 1994). The explicit element of some knowledge consists of the information or events that the individual reacts to and, therefore, can identify and express in words and numbers (Nonaka 1994; Polanyi 1966). Meanwhile, the tacit element of that knowledge is the awareness of the particulars of that information or event, which gives the context and influences how the individual anticipates, interprets, and reacts (Polanyi 1966). In other words, these particulars are only known in the context of that information or event and therefore cannot be codified as the explicit element of the knowledge. Explicit knowledge can be codified and even shared through an information technology (IT) system (Gonzalez and Martins 2014; Lee and Yang 2000).

To give an example, a KW can leave a meeting with a customer and write internal meeting notes for his/her team that the customer liked specific features (explicit knowledge), but if you ask him/her how he/she knows that the customer liked the features, he/she could only vaguely tell why he/she came to that conclusion (tacit knowledge). The KW would have relied on his/her awareness of particulars such as his/her interpretation of the body language of the customer or of some of the questions or comments the customer made. This interpretation is influenced by the KW's perceptions, which stem from his/her previous experiences, beliefs, and perspective (Nonaka and Ryoko 2003).

Figure 2 shows the four modes of knowledge conversion of Nonaka (1994). He assumed that knowledge is created through the conversion of tacit and explicit knowledge. The four modes are externalization, internalization, socialization, and combination.

Externalization is the conversion of tacit knowledge into explicit knowledge through a process that reveals hidden tacit knowledge, allowing the KW to articulate it as explicit knowledge and express it as information. Internalization is the conversion of explicit knowledge into tacit knowledge through application in relevant situations. Socialization is the conversion of tacit knowledge in the social environment into tacit knowledge of the individual through action or perception. Combination is the creation of new explicit knowledge by sorting, adding, recontextualizing, or recategorizing explicit knowledge (Nonaka 1994; Nonaka and Ryoko 2003). 
These four modes of conversion will be used in the discussion on how the KW works with knowledge. The main purpose of a KW is to acquire, create, share, or apply knowledge in his/her job to create value. Figure 3 shows important factors pertaining to how the KW acquires, creates, or applies knowledge, while Figure 4 focuses on knowledge transfer, which is the basis of sharing knowledge.

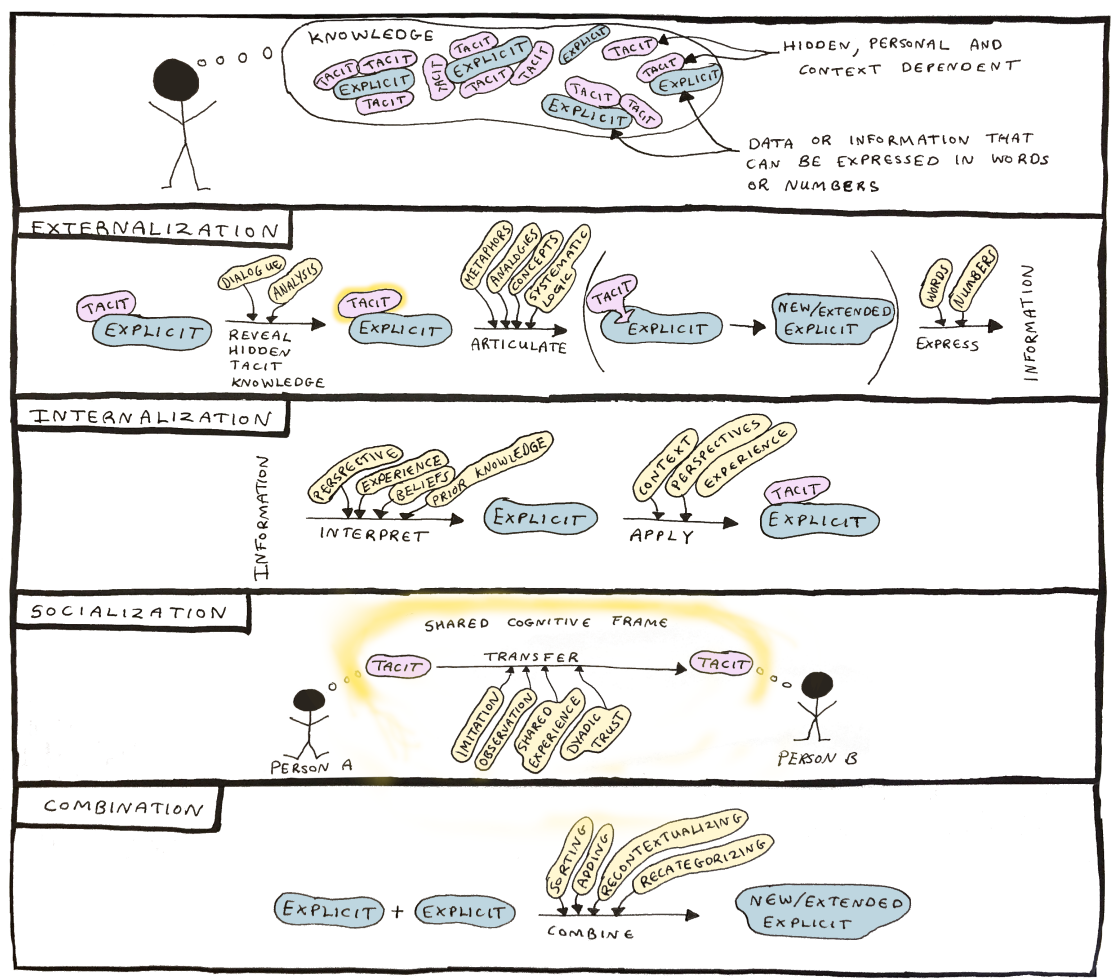

Figure 2. The four modes of knowledge creation of Nonaka (1994).

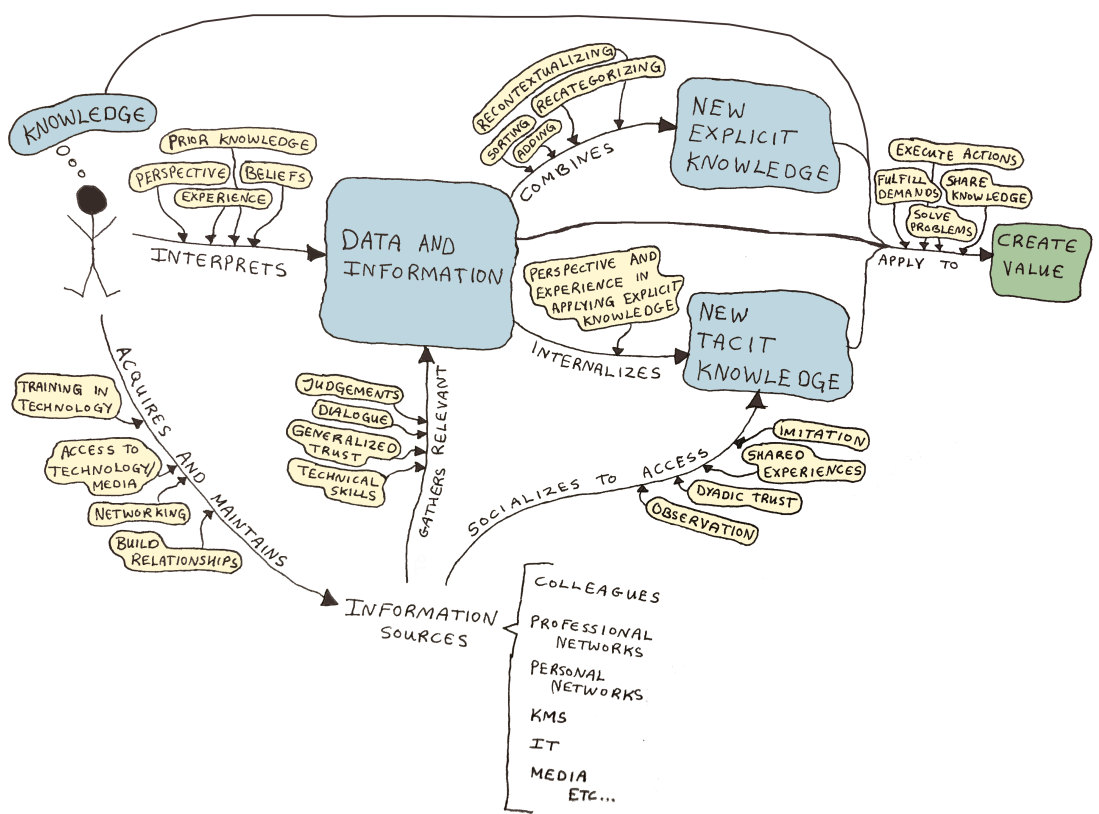

Figure 3. Acquire, create, and apply knowledge. 


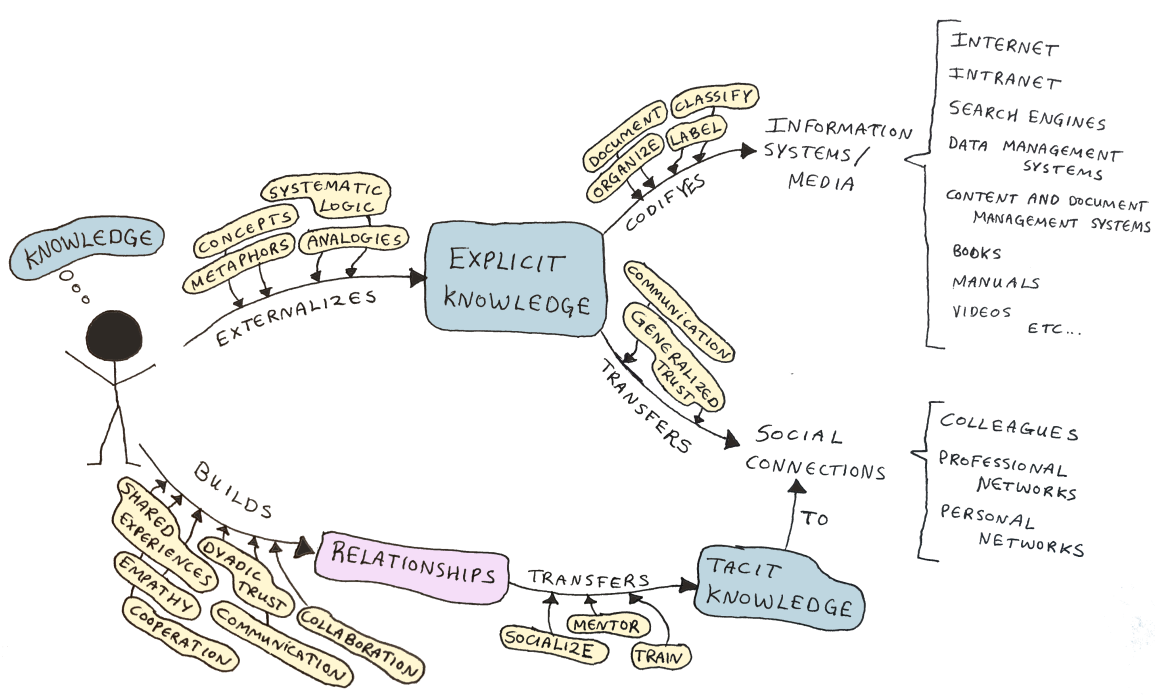

Figure 4. Knowledge transfer.

As mentioned above, the KW interprets data and information using his/her perspective, prior knowledge, experience, and beliefs. Data and information can be gathered, interpreted, and reconfigured by sorting, adding, recategorizing, or recontextualizing to create new explicit knowledge. This is the process of combination from Figure 2 (Nonaka 1994).

Data and information can be converted into tacit knowledge by individuals through internalization (Nonaka 1994). In the internalization process, the KW creates new tacit knowledge by interpreting the explicit knowledge using his/her perspective and experience in applying the explicit knowledge. The new tacit and explicit knowledge acquired or created in these processes can then be applied along with prior knowledge, data, and information to create value by allowing the KW to fulfill demands, execute actions, or solve problems that depend on the knowledge. The KW should also share his/her newly found knowledge to create value by contributing to the organizational knowledge base.

The KW needs to gather data and information to acquire or create the knowledge he/she needs to be able to execute these value-creating actions effectively and efficiently. Information can come from colleagues, professional or personal networks, technology, media, etc. The KW needs to know what information sources are available, as well as how to acquire, access, and maintain them.

The KW should be selective in the information he/she gathers since assimilating information and creating knowledge takes time and effort. In some instances, the KW could create more value by delegating an action to someone who already has the knowledge rather than trying to internalize it himself/herself. Developments in information technologies have drastically increased the availability of information (Edmunds and Morris 2000). The KW needs to be aware that not all information is created equal and should use his/her judgments to discern useful and relevant information.

Most organizations use information and communication technology (ICT) systems to store and distribute their data and information (Mládková 2011), for example ICT systems such as data management systems, content and document management systems, intranet solutions, search engines, workflow management systems, business intelligence systems, groupware, and so on (Maier 2007). The usefulness of such systems is dependent on the KWs themselves, whether they keep them up to date with relevant data and information and actually use them as information sources. It is, therefore, important that the KW be aware of these systems as potential information sources and receive training on how to codify and retrieve data and information from them.

Social relations are considered the most efficient means for transferring both tacit and explicit knowledge between individuals (Kang et al. 2007). This means that appreciating information sources is inherently understanding the social constructs within the organization 
or expert communities in which the KW is. Different types of social connections give access to different information sources. Strong and dense social connections, built on dyadic trust through direct personal experiences, are great sources for exploitative learning, which involves refining and deepening existing knowledge. This is because shared experiences are the key to transferring and acquiring tacit knowledge (Nonaka 1994). Individuals need to have a shared cognitive frame of reference to recognize, understand, and exchange tacit knowledge (Kang et al. 2007). This allows individuals to share each other's thinking processes through observation, imitation, and practice (Nonaka 1994).

This means, to access information or knowledge from colleagues or other members of an appropriate expert community, building relationships is key. The KW can build relationships and access information or knowledge by socializing, cooperating, collaborating, and communicating. Communication can take place either using face-to-face communication or using communication technologies such as email, messaging platforms, social media, telephones, or web conferencing platforms.

Networking is a way to acquire new information sources. It is a set of behaviors designed to build informal interpersonal relationships to exchange affect, information, benefits, and influence (Michael and Yukl 1993). Today, much networking happens in the virtual world of the Internet or web through social media instead of face-to-face. Social media refers to applications or web sites that enable users to exchange information and provide tools to support the maintenance of relationships, aid in the discovery of potential relationships, and the conversion of potential ties into weak and strong ties (Shalini et al. 2018; Van Zyl 2009).

Weak and nonredundant social connections, built on generalized trust accorded to the $\mathrm{KW}$ because he/she is a member of the same social unit, are great sources for explorative learning. Explorative learning involves the pursuit of knowledge that differs from the status quo and does not exist in the organization (Kang et al. 2007). In weak and nonredundant social connections, a dialogue needs to take place to convert the tacit knowledge of the parties into explicit knowledge through what Nonaka (1994) called the externalization process. Since the parties do not have shared experiences, they need to use metaphors and analogies to articulate their own perspectives and reveal hidden tacit knowledge. Through successive rounds of meaningful dialogues, concepts are created with consistent and systematic logic. This results in explicit knowledge that can be codified, communicated, and learned by others.

Figure 4 shows important factors connected to knowledge transfer that have been discussed above. The KW can create value by being an information source to others and take time to share his/her knowledge, both explicit through codification and tacit through social interactions such as socializing, mentoring, and training.

Most KWs are interdependent, that is their actions are dependent on the actions of others. This interdependence can emerge from the complexity of tasks, where the KW does not have all the knowledge or skills needed to execute them, or from the design of work processes, where the tasks are serially linked (Rosendaal 2009). Another type of interdependence is when the KWs are working towards group goals or provided with group feedback (Van der Vegt and Van de Vliert 2002).

To create a relatively permanent competitive advantage for the organization, individual learning needs to be transformed into organizational learning (Urbancova et al. 2016). Organizational learning is the development of collective insights, knowledge, and associations between past actions and future actions (Fiol and Lyles 1985; Popper and Lipshitz 1998; Urbancova et al. 2016). It is not simply the sum of each member's learning even though organizations only learn through the experience and actions of individuals (Fiol and Lyles 1985; Popper and Lipshitz 1998).

Organizational learning creates the shared mental models, norms, and values over time that allow for the transfer of tacit knowledge (Fiol and Lyles 1985; Popper and Lipshitz 1998). These shared mental models, norms, and values are transmitted to present and future employees through the organizational culture and learning systems. By sharing 
knowledge acquired while executing actions, the KW contributes to the learning systems of the organization. Until KWs share both their tacit and explicit knowledge within the organization, it is the individual's capital, not the organization's, and is therefore an unvalued asset by the organization (Bogdanowicz Maureen and Bailey Elaine 2002).

As is apparent in the discussion above, working with knowledge is very complex and depends on many factors. Such as the organizational environment, culture, the perspectives of individuals, social connections between individuals, and the availability of technology, information, and data. This complexity means that there are many fields and disciplines dealing with knowledge (Maier 2007). From these fields, the multidisciplinary field of knowledge management (KM) emerged, which is based on the belief that "there are substantial benefits to be gained from the systematic and conscious treatment of knowledge-related processes in organizations" (Maier 2007, p. 59). Many organizations have implemented KM initiatives to improve their way of handling knowledge to improve organizational performance (Maier 2007).

Knowledge management systems (KMSs), which rely on modern ICTs, are used as enablers of KM within organizations (Oyefolahan and Dominic 2013). They combine and integrate services for the handling of explicit and tacit knowledge (Maier 2007). KMSs facilitate, for example, the storing, organizing, distributing, and retrieval of explicit knowledge. They also provide services for the handling of tacit knowledge, which ease the identification of experts within the organization, the creation of communities of experts, and collaboration. Most larger organizations have some sort of KMS that the KW needs to be aware of and utilize to access information sources and as an information source itself.

The success of a KMS is dependent on the willingness of employees to use it, the willingness to codify their own knowledge into the system, acquire knowledge from the system, and create new knowledge through combination and collaboration with others (Oyefolahan and Dominic 2013). Factors such as training in the KMS technology, as well the as ease-of-use and quality of the KMS affect whether the KMS is adopted by the KWs in the organization (Kundapur and Rodrigues 2012).

With these emerging KMSs, ICT systems, and media outlets, such as the Internet, there is an abundance of information. It is, therefore, important to use discernment when choosing information sources and what information to process. This abundance of information may lead to information overload or analysis paralysis. Then, the KW cannot process all the information efficiently, leading to omissions, errors, and stress (Edmunds and Morris 2000). Information overload or analysis paralysis affect not only decision-making, but have a depleting effect on personal resources. Hemp (2009) stated "the stress of not being able to process information as fast as it arrives-combined with the personal and social expectation that, say, you will answer every e-mail message-can deplete and demoralize you". The next section delves into the KW's personal resources and how they are depleted and restored.

\subsection{Personal Resources}

Hobfoll et al. (2003) defined personal resources as aspects of the self that are generally linked to resiliency. They refer to an individual's sense of his/her ability to control and impact his/her environment successfully. Resiliency is the capacity to recover quickly from stress. These are resources such as self-efficacy, sense of mastery, optimism, characteristic degree of goal pursuit, social support, and self-esteem. Kira et al. (2010), on the other hand, defined personal resources as "mental abilities and states, such as knowledge and selfesteem", as well as "dispositional orientations or habitual behaviors, such as a preferred coping strategy or preventive behavior in maintaining one's health". Greenblatt (2002), meanwhile, defined personal resources as the physical, psychological, cognitive, and social resources at the disposal of an individual, which "provide the fuel necessary to engage with and accomplish all of life's activities".

Physical resources come from our body. They consist of our physical strength, endurance, and reactions to physical stimuli. Psychological resources refer to the individual's 
affective capabilities, emotional intelligence, and responses to emotional stimuli. They include faith as well, which comes from the individual's connection to deeply held values, purposes, and vision. Cognitive resources are natural and learned intellectual capacities, the ability to access them, and the energy necessary to use them. Social resources include the capabilities to interact and connect with others, as well as the ability to access social resources for personal benefit.

The perspective of personal resources of Greenblatt (2002) seems to be different from the other definitions, but after a closer comparison, it becomes clear that these four groups of personal resources all encompass aspects of the self that are linked to resiliency. Figure 5 shows how these three definitions all refer to personal resources as aspects of the self linked to resiliency.

In Figure 5, resiliency seems to have a simple relationship with the personal resource reserves where resiliency lowers if the personal resource reserve decreases. This is a simplification of this relationship. According to Cake et al. (2017), resiliency is a dynamic process in which personal resources and contextual resources interact over time through particular strategies to enable sought-after mental states, such as enjoyment, self-esteem, professional engagement, satisfaction, well-being, and so on. Contextual resources include, for example, someone's social support, relationships, feedback, decision latitude, skills, discretion, and culture. Strategies, however, are the dispositional orientations and habitual behaviors that someone uses to try to restore their personal resource reserves, such as sleep, meditation, time management, nutrition, humor, and so on.

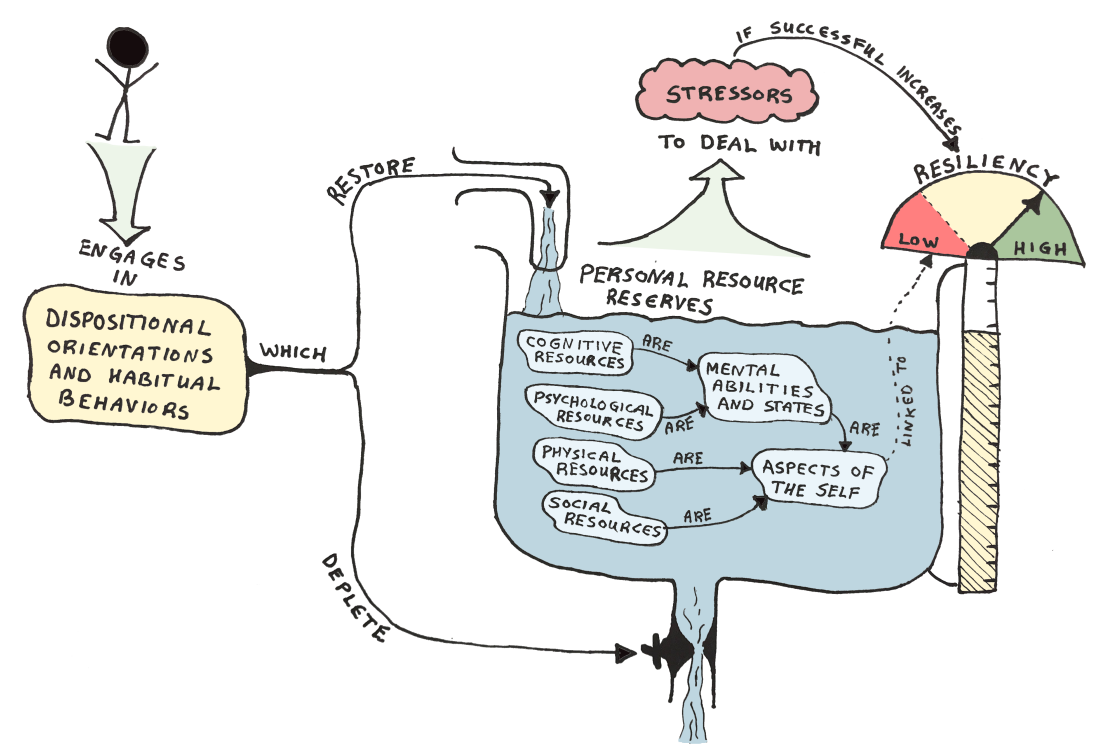

Figure 5. Definition: personal resources are aspects of the self linked to resiliency.

The KW's personal resources are subjective and influenced by the KW's perceptions of himself/herself and the world. This means that perceived personal resources change over time with the ever-changing worldview of the KW, in different situations and environments. The assessment of what personal resources are available and the threat of loss, actual loss, or resource gains usually happens subconsciously, causing habitual responses to different stressors and challenges in the environment. How an individual responds is a function of his/her personality, constitution, perceptions, and the context in which the stressors occur (Hobfoll 1989).

Habitual responses to offset resource loss are often called coping strategies or behaviors (Hobfoll 1989). When reacting to a loss of a resource, individuals make use of other resources that they possess, which can result in a depletion of those resources. When choosing to utilize a given coping strategy or behavior, the individual subconsciously evaluates his/her potential losses, determines what he/she loses by expending other resources, and 
analyzes the chance of success. Circumstances that threaten an individual's status, position, economic stability, loved ones, health, basic beliefs, or self-esteem are common causes of personal resource depletion. Events that clearly reflect loss are the most psychologically threatening such as being fired, retirement, foreclosure, divorce, or death (Hobfoll 1989).

These kinds of big events are not needed for a knowledge worker to feel chronic stress that affects his/her performance. Multiple personal resources are used to accomplish tasks, and the tasks have a different effect on different personal resources (Greenblatt 2002). A task may have a depleting effect on one personal resource, but a restorative effect on another. Executing many tasks in a row using behaviors or in conditions that have a depleting effect on a specific personal resource will lead to chronic stress that undermines performance (Greenblatt 2002; Loehr and Schwartz 2001).

There is a resiliency threshold. If the personal resources are depleted below it, the typical impact of depleting behaviors or conditions are magnified and the impact of restorative behaviors or conditions are reduced. This is typically the cause of the exhaustion or burnout of KWs, a series of small personal resource losses that are insufficiently offset by personal resource gains, eventually using up the personal resource reserves necessary for successful coping strategies or behaviors. Figure 6 shows the interaction between the personal resource reserves and the effects of engaging in certain behaviors or conditions.

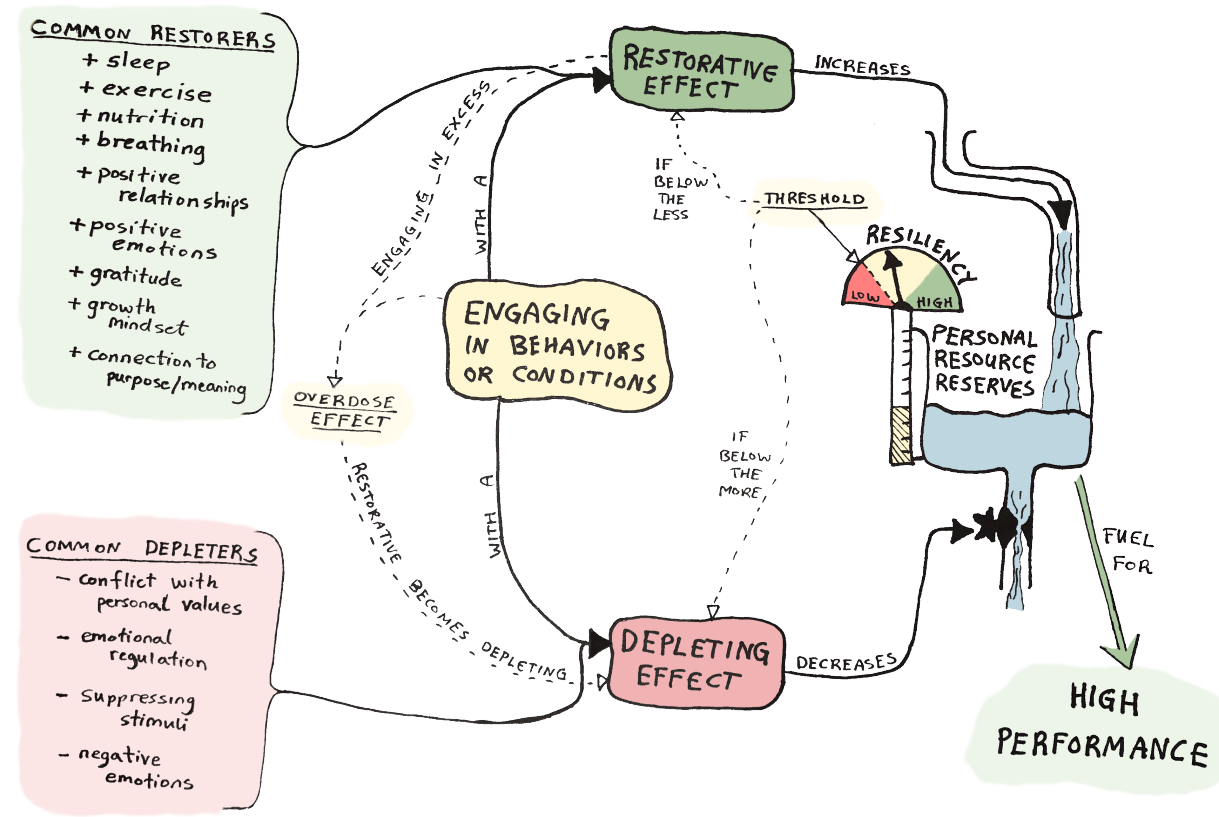

Figure 6. Personal resource reserves.

The KW's wellbeing and performance, therefore, can be improved by choreographing tasks to optimize the use of the KW's personal resources (Greenblatt 2002; Loehr and Schwartz 2001), that is to make sure that depleting behaviors and conditions are offset by restorative behaviors and conditions. It is not only important to balance depletion and restoration. The personal resource reserves can also be expanded by engaging in restorative actions more frequently or in actions that have a larger restorative impact. The KW needs to use self-awareness to identify his/her personal resources, his/her personal resource reserve levels, and the different effects his/her behaviors and the conditions he/she is in have on them. With this awareness, the KW can sequence his/her tasks strategically to positively impact resource levels (Greenblatt 2002).

There are though some common behaviors and conditions that are restorative for most people such as sleep, exercise, nutrition, breathing, positive relationships, positive emotions, gratitude, a growth mindset, and connecting to your purpose or meaning (Fritz et al. 2011; Greenblatt 2002; Loehr and Schwartz 2001; Peseschkian and Remmers 2020). 
Greenblatt (2002) found in her research that a KW can experience what she called an overdose effect, when characteristically restorative behaviors or conditions become depleting when experienced in excess. Common behaviors and conditions that are depleting for most people are conflicts with personal values, emotional regulation, suppressing stimuli, and negative emotions (Greenblatt 2002; Loehr and Schwartz 2001).

\subsection{Competencies}

A competency is a set of behavior patterns organized around intent (Boyatzis 2008). Intent is the force that causes action toward an outcome (Spencer and Spencer 1993). This means that competencies both imply what a KW can do and what he/she wants to do (Ryan et al. 2009). Competencies allow the KW to demonstrate behavior patterns that lead to or cause effective or superior performance (Boyatzis 1982).

A competency may exist within the KW at various levels. Boyatzis (1982) defined three levels of a competency: (1) the subconscious level of motives and traits, (2) the conscious level of self-image and social role, and (3) the behavioral level of skill. Spencer and Spencer (1993) presented these levels in an iceberg model, where the visible components of a competency, skill and knowledge, stood up above the waterline, while the hidden components, self-image, traits, and motives, were under the water. The definitions of these components are (Boyatzis 1982; Spencer and Spencer 1993):

1. Motives are recurrent thoughts about a goal state or situation that drive an individual to a certain behavior;

2. Traits are a characteristic way an individual responds to certain stimuli, that is the consistent responses he/she has to similar situations or information;

3. Self-image is an individual's perception of himself/herself and the interpretation and labeling of that image in the context of values, both his/her own and the values in the environment;

4. Social role is an individual's view of how he/she fits in with regard to the characteristics he/she possesses and the interpreted expectations of others;

5. Knowledge is the internalized information an individual has in specific content areas;

6. Skill is the individual's ability to demonstrate specific behavior patterns that are functionally related to attaining a performance goal.

Figure 7 shows these components in an extended version of iceberg model of Spencer and Spencer (1993). The figure shows how the KW's characteristics and environment affect these components and how the components lead to effective or superior performance.

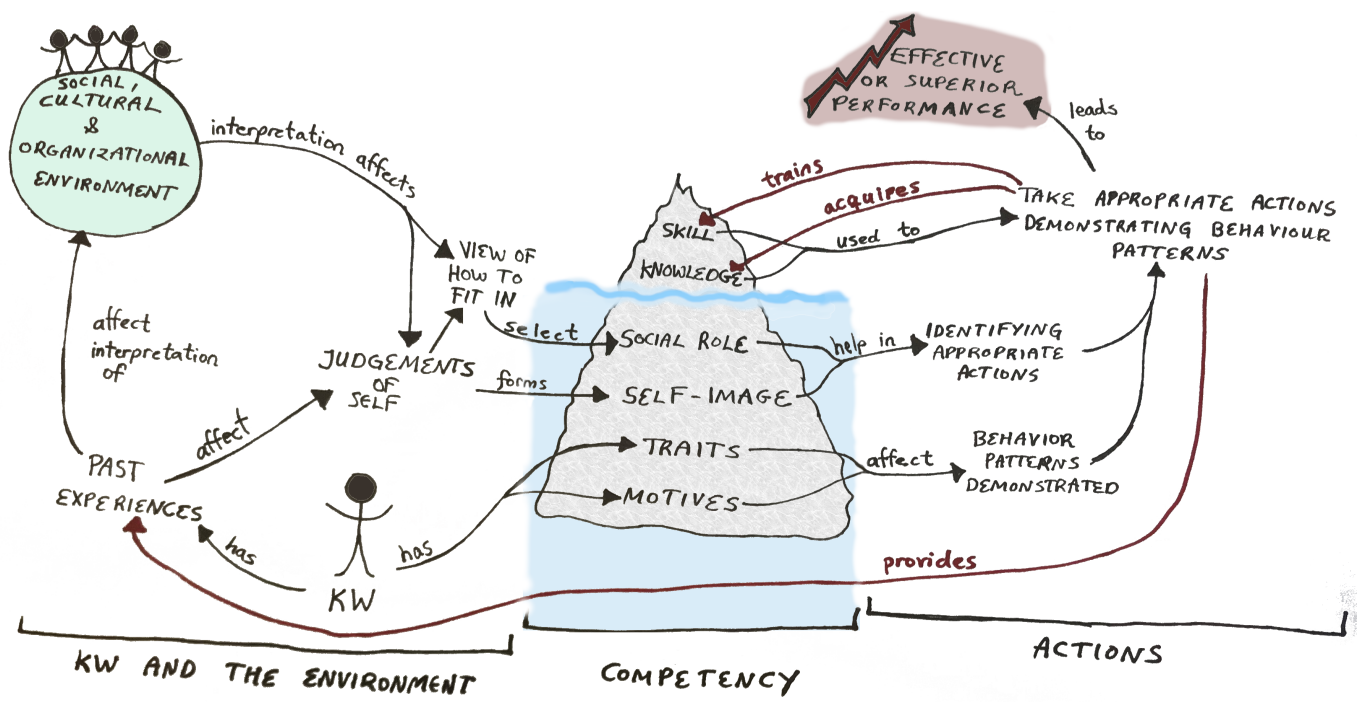

Figure 7. Levels of a competency, what affects them, and how they contribute to effective or superior performance. 
It is best to read the figure from left to right starting at the KW stick figure. The KW has the underlying characteristics of motives and traits. The KW has past experiences, as well, that affect his/her interpretation of the social, cultural, and organizational environment. His/her past experiences and his/her interpretation of the environment affect his/her judgments of self, which form his/her self-image. His judgments of self and his/her interpretation of the environment affect how he/she views how he/she fits in, which influences what social role he/she selects in the situation.

The four components of a competency that are hidden under the water in the iceberg model control the surface behaviors (Garavan and Mcguire 2001). Traits and motives are at the base of the iceberg and are difficult to assess and develop (Spencer and Spencer 1993). They affect what behavior patterns are demonstrated. A KW will, therefore, be unsuitable for an organizational role if the $\mathrm{KW}$ lacks the traits and motives for the required competencies. This is the basis of the popular saying: hire for attitude or character and train skill.

Social role and self-image are a bit higher up, but still hidden under the waterline. They can be changed by training, psychotherapy, and positive developmental experiences (Spencer and Spencer 1993). They help in identifying the appropriate actions to take for effective or superior performance. Skill and knowledge are at the top of the iceberg, fully visible above the waterline. They are relatively easy to develop. Skill and knowledge are used to take appropriate actions, demonstrating specific behavior patterns that lead to effective or superior performance. By taking the appropriate actions, knowledge is acquired and the ability to demonstrate these specific behavior patterns improves, which effectively trains the skill. The collection of actions taken by the KW over time provide the past experiences of the KW.

Boyatzis (2008) identified three clusters of competencies connected to outstanding performance from research published in the last thirty years:

1. Cognitive intelligence-competencies that relate to "the ability to think or analyze information and situation" (p. 8), for example systems thinking and pattern recognition competencies;

2. Emotional intelligence-competencies that relate to "the ability to recognize, understand and use emotional information about oneself" (p. 8), for example self-awareness and self-management competencies;

3. Social intelligence-competencies that relate to "the ability to recognize, understand and use emotional information about others" (p. 8), for example social awareness and relationship management including empathy and teamwork competencies.

The KW needs to appreciate his/her own competencies when discerning what to do and how to do it. To appreciate his/her own competencies, the KW needs to acquire and develop the competency of self-awareness. Hall (2004) called it a metacompetency since it helps the individual learn how to learn new competencies. Self-awareness refers to how conscious the individual is of his/her self-image, own inner state, and his/her impact on others. Through his/her self-image, the individual perceives his/her particular set of competencies from his/her experiences, interests, values, his/her belief in his/her capabilities to perform certain tasks, and feedback from others (Hall 2004).

There is a vast amount of literature on factors that influence the work of the KW. This literature review focused on what is value in knowledge work, competencies, knowledge, and personal resources. No papers were found that connected all these concepts together to create a holistic view of the activities of the KW when creating value. This paper attempted to do just that. The next section uses the knowledge gained from the literature review to identify activities and develop a purposeful activity model of a system for the individual.

\section{Purposeful Activity Model of a System for the Individual}

The first step in the soft systems methodology (SSM) is to analyze the problem situation and define relevant systems from different viewpoints of stakeholders. These different viewpoints are called problem owners. A problem owner is someone who experiences 
unease about a situation, is affected by it, and feels that it can be improved (Checkland 1993). The second step in the SSM is to formulate purposeful activity models for relevant systems for each problem owner. Purposeful activity models are a tool in the SSM. The objective of the SSM is not to draw up an accurate representation of the real world, but to structure an exploration of it as a learning system using systems thinking (Checkland 2000).

Óskarsdóttir and Oddsson (2017) executed two literature reviews to explore the problem situation of managing and improving knowledge worker productivity (KWP). They defined two relevant systems for two problem owners, the individual knowledge worker (KW) and the organization. This section goes into detail about the development of a purposeful activity model for the system, defined by Óskarsdóttir and Oddsson (2017), owned by the individual KW, and presents it.

\subsection{Developing the Purposeful Activity Model}

The SSM defines systems using root definitions that describe them as transformation processes. Purposeful activity models are, therefore, developed by identifying and linking the activities relevant to acquiring the input, transforming the input into output, and generating target outcomes (Checkland 2011). According to Zwikael and Smyrk (2012), outputs are tangible artifacts produced from the work of the transformation process. However, in the context of knowledge work, defining the outputs as tangible artifacts is too narrow. Outputs in knowledge work can be tangible, for example documents or products, but also intangible, such as services or knowledge. Let us extend the definition of output to include both tangible and intangible artifacts. Target outcomes, on the other hand, are intangible desired end-effects that arise when the output from the transformation process is utilized (Zwikael and Smyrk 2012).

Óskarsdóttir and Oddsson (2017, p. 18) defined the system for the individual KW as "a system, owned by the individual, which transforms perceived effort of the individual knowledge worker into perceived value by the organization by managing personal resources, being effective and efficient". This root definition was found to be too restrictive when developing the purposeful activity model. It does not include the conflict of interest that the KW needs to deal with when creating value. He/she needs to interpret what is value and choose whether to create value for himself/herself, for the organization, or for someone in his/her social system. The root definition above assumes that the KW can interpret effectively what the organization perceives as value and that the KW's objective is to only create value for the organization. A new root definition was formulated as seen in Table 1.

Table 1. Root definition of a system owned by the individual knowledge worker (KW).

\section{Root Definition:}

A system, owned by the individual KW, in which the KW uses resources to execute actions exerting effort to create tangible or intangible artifacts with the intention of creating value.

Figure 8 maps the root definition to the ITO model of a transformation process, where input is transformed into output, which generates target outcomes (Zwikael and Smyrk 2012).

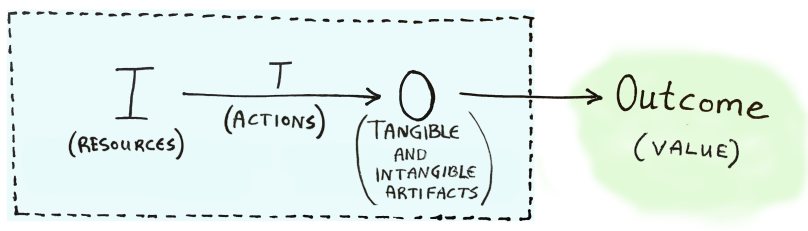

Figure 8. ITO model. 
As seen in Figure 8, the inputs (Is) are resources. Resources can be anything the $\mathrm{KW}$ uses to execute actions to create artifacts, for example tangible resources such as office supplies, documents and technology, as well as intangible resources such as time, knowledge, competencies, and personal resources. Since the focus of this purposeful activity model is on the individual KW in the situation of managing and improving KWP, only resources that influence decision-making that affects KWP were explored. From the literature review, seven activities were identified that were needed to obtain the input, which were assembled into two groups, awareness and personal aspects.

In the awareness group are the activities: appreciate what is value, appreciate competencies, appreciate information sources, and appreciate personal resources. The word appreciate is used because it encompasses the importance of both fully understanding and recognizing the worth of these aspects in the awareness group. In the personal aspects group are the activities: acquire and develop competencies, acquire and maintain information sources, and manage personal resources.

The transformation $(\mathrm{T})$ consists of the actions executed by the KW to transform the resources to the output $(\mathrm{O})$ of tangible and intangible artifacts. In other words, the outputs (Os) are the results of the actions the KW takes. Five activities were identified that the KW needs to perform to execute actions with the intention of creating value. The activities were put together into the group actions and are: identify actions that contribute to value creation, evaluate the competencies and knowledge needed for actions, evaluate the effort needed to execute the actions, select actions, and execute actions exerting effort.

The targeted outcome is value. Value is subjective, so what is value is open to interpretation. To be able to reach a target outcome, the output needs to be delivered to someone who then utilizes it in a way that generates the target outcomes (Zwikael and Smyrk 2012). Three activities were identified that are needed to deliver the results of the KW's actions. They were put together into the group value contribution and are: communicate the results of actions to relevant parties, share knowledge acquired while executing actions, and evaluate whether actions created value.

The next section presents the purposeful activity model where the identified activities are linked logically together. Delving deeper into these activities and how they are interdependent gives a structure to our exploration of what influences the productivity of the KW at work. Figure 9 shows the four groups of activities identified and how they are linked.

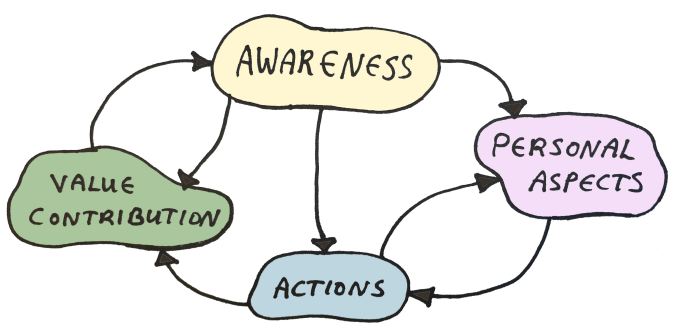

Figure 9. The four groups of a system for the individual.

The arrows show what groups and activities depend on each other. The activities in the personal aspects group depend on the activities in the awareness group. The arrow, therefore, points from awareness to personal aspects. The actions group uses awareness and personal aspects to execute actions, but acquiring and maintaining personal aspects takes place during the execution of actions. That is why there are arrows in both directions. Value contribution depends on the outcomes of actions and uses awareness to evaluate the value created, which in turn informs the KW. Awareness, therefore, also depends on activities in the group value contribution. These links will become clearer in the review of the purposeful activity model in the next section. 


\subsection{Purposeful Activity Model}

The purposeful activity model of a system for the individual shows the activities the individual KW goes through when creating value and how they are linked. A KW goes through many of these activities subconsciously, making split-second decisions based on intuition. If a KW skips some activities or carries them out with the wrong assumptions, this could lead to less effective decisions or unnecessary effort, which can affect his/her productivity, for example wasting effort on actions that do not create value, executing actions using too much effort that are better suited for others, or delivering results of actions to irrelevant parties or even not at all.

Figure 10 shows the purposeful activity model of a system for the individual with the identified activities in the four groups: awareness, personal aspects, actions, and value contribution.

To execute appropriate actions, the KW needs to identify actions that contribute to value creation. This activity is at the top of the actions group in Figure 10. Every job is full of demands. The KW is inundated with demands from himself, the organization, and others in his/her social system. Nevertheless, being productive is not about blindly reacting to demands and getting things done, regardless of whether fulfilling the demands creates value or not.

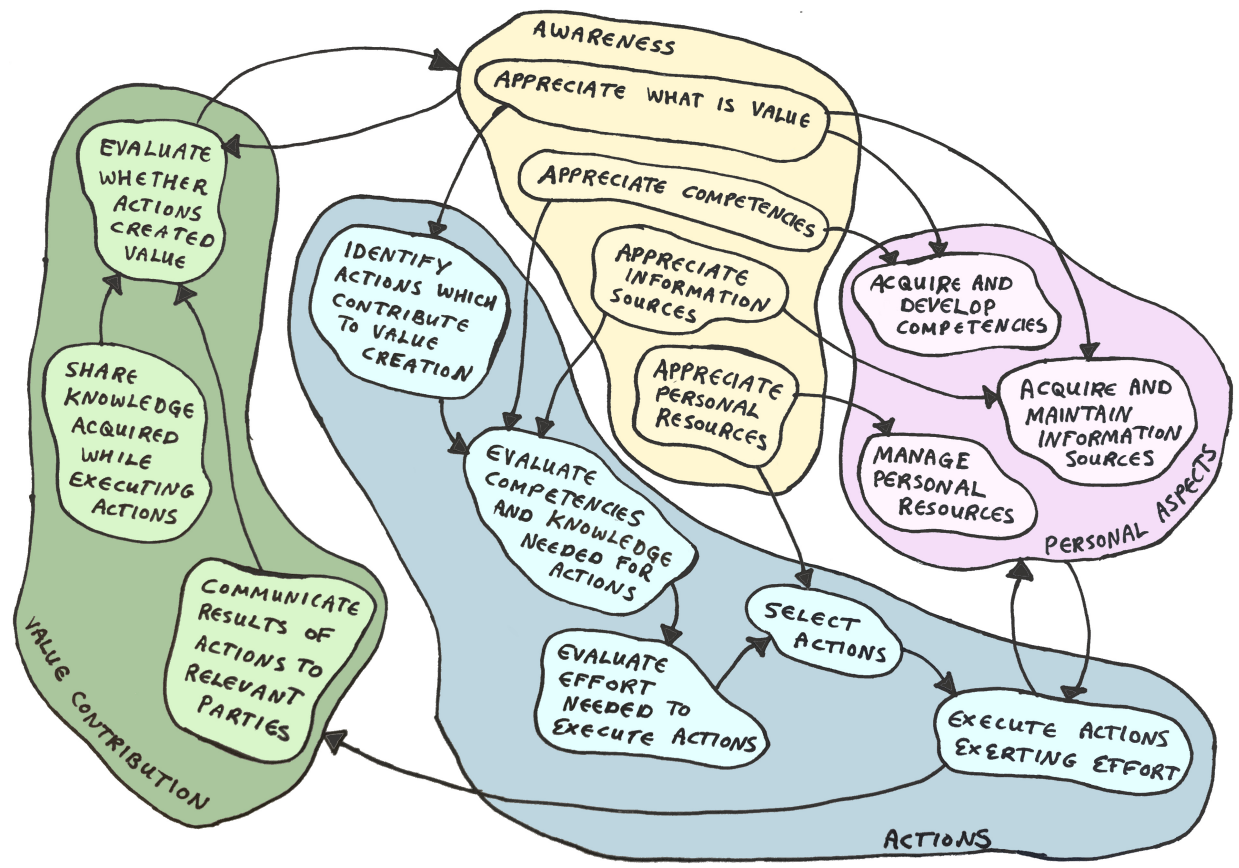

Figure 10. Purposeful activity model of a system for the individual.

If the KW binds emotionally or intellectually to a demand, it becomes a commitment. Too many commitments can overwhelm the KW by pulling at his/her attention. This has a depleting effect on his/her personal resource levels, causing undue stress. Under chronic stress, the KW needs to exert more effort when executing actions. The KW needs to appreciate what is value to be able to identify worthwhile actions from the constant demands on him. Appreciate what is value is the activity on top of the awareness group.

The KW appreciates what is value through self-awareness, socialization, and organizational socialization. The KW identifies his/her own needs, in other words what he/she finds valuable, using self-awareness. Socialization and organizational socialization are processes in which a KW adapts from an outsider to an integrated and effective insider. These processes give a group of people a shared cognitive frame built on shared experiences, trust, imitation, and observation. This allows the KW to interpret more successfully what the organization or others in his/her social system perceive as value, making it more likely that the KW creates the value he/she intends. 
Appreciating what is value also touches on the evaluation of whether it is appropriate to execute actions that create known value or innovating actions that might or might not create value. Innovation is an investment in possible future value and is inherently risky. However, successful innovations can be the key to competitive advantage and human progress. Section 2.1 went into detail about factors that influence the appreciation of value extracted from the literature review.

The KW needs to evaluate competencies and knowledge needed for actions so he/she can evaluate the effort needed to execute actions, the next two activities in the actions group. To be able to evaluate competencies and knowledge needed for actions, the KW needs to appreciate competencies and appreciate information sources, the next two activities in the awareness group.

It is not enough to be aware of what actions contribute to value creation; the KW also needs to appreciate his/her own competencies to know where he/she has the unique ability to contribute. To appreciate his/her own competencies, the KW uses self-awareness. The individual perceives his/her particular set of competencies through the lens of his/her experiences, interests, values, his/her belief in his/her capabilities to perform certain tasks, and feedback from others. He/she must identify whether he/she needs to acquire new competencies to create value, develop the competencies he/she already has, or transfer into a more suitable job or role.

That brings us to the first activity in the personal aspects group, acquire and develop competencies. Different jobs and roles within organizations need different competencies. Some competencies can easily be acquired and developed, but others are more inherent, and the lack of them could make the KW unsuitable for some roles. A common example is of the highly valued expert that is promoted to a management role in which he/she becomes unsuccessful because he/she is lacking fundamental competencies needed for management, such as leading others and interpersonal understanding. Section 2.4 describes the components of competencies that affect how much effort it takes to acquire and develop them.

The KW seldom has all the information and knowledge required for an action. After the KW has discerned what he/she requires, the KW needs to appreciate information sources. Appreciating information sources is necessary to find and gather the required information or knowledge. It is important to put effort into acquiring and maintaining information sources to improve access and availability to information and knowledge. Acquire and maintain information sources is the second activity in the personal aspects group.

Information and knowledge can come from colleagues, a professional or personal network, technology, media, books, and so on. If the KW needs to acquire much new information or knowledge to execute an action, it might not be worth the effort and better to delegate to someone already having the knowledge. Social relations are considered the most efficient way to transfer knowledge. Acquiring and maintaining information sources is, therefore, mostly about obtaining new social connections and building relationships. Networking is a way to acquire new information sources through social connections, and building relationships is about creating shared experiences. Shared experiences allow individuals to have a shared cognitive frame of reference to recognize, understand, and exchange knowledge. Different types of social connections give access to different types of information or knowledge. Appreciating information sources is also about being aware of information communication technology (ICT) systems as potential information sources and acquiring the skills to utilize them. Section 2.2 explained factors that influence how the KW works with knowledge.

These are the factors the KW needs to have in mind when evaluating the effort needed to execute actions, the third activity in the actions group. KWs use their intuition, prior experience, self-awareness, and cues from their environment to perform this evaluation. KWs do not perform this evaluation consciously unless they need to for planning reasons. An action that requires developing new competencies or gathering much information might not be the most efficient choice, but might be an effective choice. For example, it might be effective if the competencies or knowledge acquired are useful for future actions. 
From the insight gained from the evaluations, the KW selects actions to execute, the fourth activity in the actions group. When selecting actions, the KW must take into account the evaluations of the effort needed and his/her personal resource levels. He/she needs to appreciate personal resources, the last activity in the awareness group. Personal resources are aspects of the self linked to resiliency and provide the fuel necessary for high performance. Section 2.3 gave examples of personal resources and described how engaging in behaviors or conditions affect the personal resource reserves.

In essence, every action the KW takes affects his/her personal resource levels. Actions can be depleting or restorative. The KW needs to be aware of his/her personal resource levels and how an action affects him/her when selecting actions to execute. The KW needs to manage his/her personal resources, the last activity in the personal aspects group. The KW should strategically sequence and time the sources of restoration and depletion of his/her personal resources for high performance.

Executing many depleting actions in a row can bring the KW's personal resource levels below a resiliency threshold. Then, the impact of depleting events is magnified, and the impact of restorative events is reduced, leading to worsening performance. This kind of chronic stress without recovery can lead to exhaustion or burnout. The KW needs to reevaluate actions regularly in regard to their effect on his/her personal resources. The effect on his/her personal resources changes over time, in different situations, at different personal resource levels, and in relation to what is going on in his/her life.

After the KW has selected actions, he/she needs to execute actions exerting effort, the last activity in the actions group. While executing the actions, the KW uses his/her personal aspects in the group. The activities in that group are executed alongside the actions. As the KW interacts within the organization or his/her social system, he/she uses his/her prior education, experience, and competencies to develop his/her personal aspects, further adding to his/her human capital and to the value of the organization.

From the actions the KW executes, tangible and intangible artifacts are created. These artifacts do not have value until they are utilized in a way that generates value for the individual KW, the organization, or others in the KW's social system. This brings us to the last group in the model, the value contribution group. There are three activities in this group; communicate results of actions to relevant parties, share knowledge acquired while executing actions, and evaluate whether actions created value.

The KW should communicate results of actions to relevant parties to generate value from the artifacts he/she creates. Most KWs are interdependent; their actions are dependent on the actions of others. This interdependence can emerge from the complexity of tasks, from the design of work processes or from group goals and group feedback. The KW needs to be aware of his/her interdependence to identify to whom he/she needs to communicate the results of specific actions. The KW's perception of the work status, expertise, and responsibilities of others will provide context to his/her actions and what results are relevant to whom.

The second activity in the value contribution group is share knowledge acquired while executing actions. By sharing knowledge, the KW contributes to the learning systems of the organization or of other groups and communities in his/her social system. It depends on the type of knowledge and what methods of sharing are appropriate. Knowledge that is personal and context dependent, tacit knowledge, needs conversations in a climate of supportiveness, belongingness, and mutual trust, while explicit knowledge can be easily codified and entered into ICT systems to be stored and managed. Until KWs share both their tacit and explicit knowledge within the organization or their social system, it is the individual's capital and is, therefore, an unvalued asset by the organization or others in the social system.

The last activity in the value contribution group is evaluate whether actions created value. Continuous self-reflection and being open to feedback from the environment promote new knowledge creation and increase the quality of the knowledge created. This kind of evaluation can be performed through monitoring or by inquiry. Monitoring is when 
the $\mathrm{KW}$ attends to naturally occurring information cues, while inquiry is when the $\mathrm{KW}$ actively generates the information cues by asking questions or initiating conversations. By evaluating whether actions created value, the KW can fine-tune his/her appreciation of what is value in the awareness group, which should lead to increasing effectiveness in the selection of actions to execute.

\section{Discussions and Conclusions}

This research used the soft systems methodology (SSM) to explore knowlegde worker productivity (KWP) and formulated a purposeful activity model of the system from the viewpoint of the individual knowledge worker $(\mathrm{KW})$. The objective was to find and define holistic systems that affect the productivity of the KW to increase our understanding of KWP, so applicable frameworks and methods can be found in future research to manage, measure, and improve it holistically. The knowledge gained from a literature review of what is value, competencies, knowledge, and personal resources was used to develop the purposeful activity model of the system from the viewpoint of the individual. The purposeful activity model is not an accurate representation of the real world, as models built for simulation, but a model of the process of how we explore the world. The purposeful activity model gives a holistic foundation to facilitate structured debates on KWP from the viewpoint of the individual, which is often overlooked in research on KWP. Most research on KWs and KWP is from the viewpoint of the organization.

The purposeful activity model views the KW's work as a process, a process where the KW uses resources (input) to execute actions to create tangible or intangible artifacts (output) with the intention of generating value (outcome). As mentioned in the Introduction, KWP can be viewed as the ratio of value created to effort exerted by the KW while executing the actions to create the value. From that proposition, it can be presumed that KWP can be improved by maximizing value creation while minimizing the effort exerted by the KW to create that value. In other words, KWP can be improved by making the transformation process more efficient. However, because of the subjective nature of both value and effort, it is not enough to focus on efficiency to improve KWP. Effectiveness, doing things right, is just as important.

Figure 11 shows an overview of the identified activities mapped to the transformation process and the implications they have for managing and improving KWP.

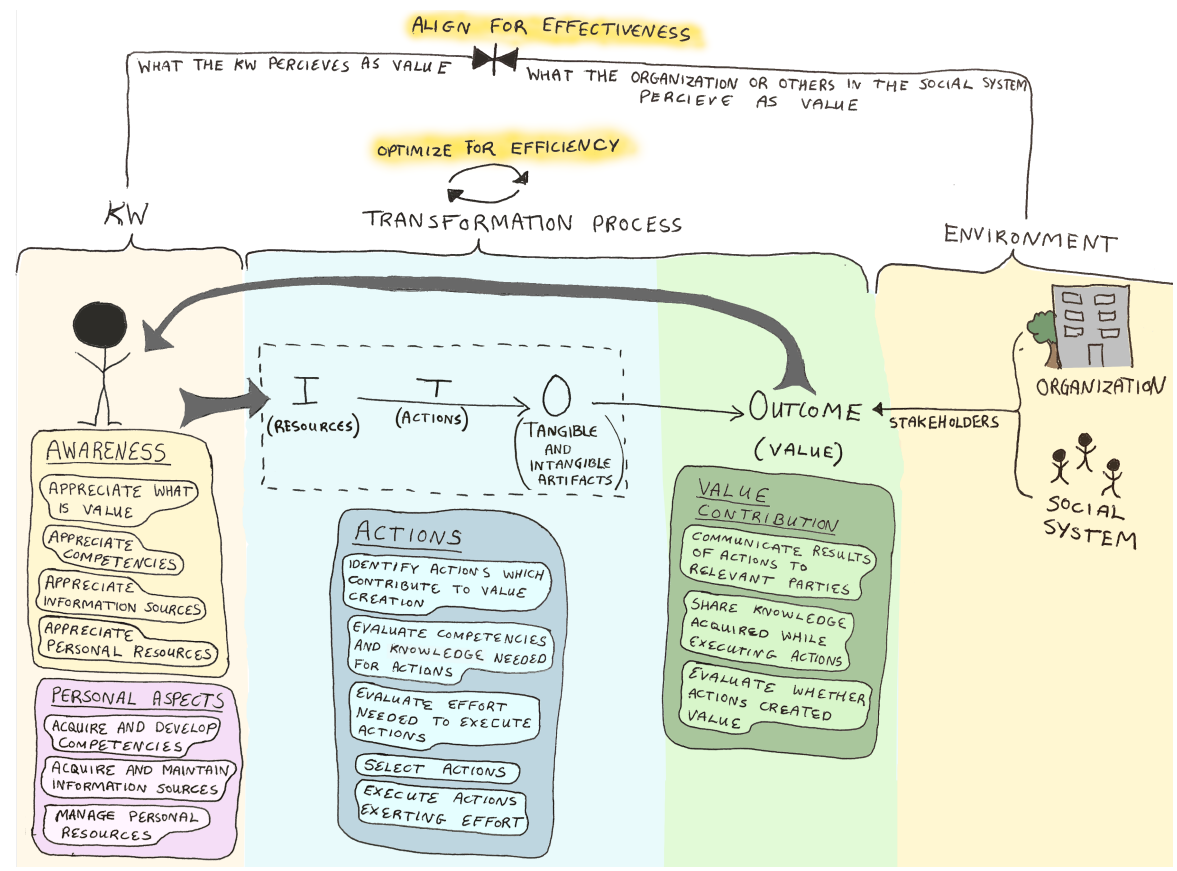

Figure 11. Transformation process of a system for the individual knowledge worker (KW). 
To be effective when creating value for others, what the KW perceives as value needs to be aligned with what the organization or others in the social system perceive as value. The KW interprets what is valuable to the organization from the organizational culture, environment, and leadership. Furthermore, he/she interprets what is valuable to others in his/her social system through their behaviors, actions, and attitudes. If there is a mismatch between the KW's interpretation and what others perceive as value, the KW might not create the value he/she intends. In other words, he/she will not be effective. The KW can fine-tune his/her appreciation of what is value by evaluating whether his/her actions created value using self-reflection and being open to feedback.

The KW identifies his/her own needs and what he/she finds valuable through selfawareness. The KW intrinsically gravitates towards actions that fulfill his/her own needs and elicit well-being. However, the organization and others in his/her social system are stakeholders of the generated target outcome, that is the value created. The organization needs to motivate their KWs to create value for them. It is, therefore, important for organizations to understand their KW's and invest in getting to know them. Subsequently, they can design the actions needed to fulfill their organizational objectives in a way that they fulfill the needs of their KW's as well.

There can also be a conflict between what others in the social system and the organization perceive as value. This conflict covers subjects such as work-family balance, the management principle of unity of command, corporate social responsibility, and so on. This will be explored further in future research where a purposeful activity model for the organization will be formulated.

The KW can be efficient by optimizing the transformation process. That includes choosing actions that maximize value creation and/or minimize effort exerted. The KW can, for example, minimize effort exerted by managing his/her personal resources. He/she can choose and sequence actions strategically to positively impact his/her personal resource levels. His/her personal resource levels affect how much effort is exerted when executing actions. The KW, therefore, needs to have the autonomy to manage the timing and sequencing of his/her own work.

Efficiency improvements can be found in most activities in the purposeful activity model, but certain actions to improve efficiency in one activity may be at the cost of the functioning of other activities or effectiveness. It is not advisable to focus too heavily on efficiency improvements. This might create a bias for choosing actions that create known value immediately or require the least amount of effort.

Sometimes, the KW can create more value down the road by investing in selfdevelopment, by choosing actions that require new knowledge or competencies, in other words choosing actions that require more effort now for value gain later. This also applies to choosing innovative actions that might or might not create value. Innovative actions, although risky, are important for human progress and the survival of organizations in the long run. Collaboration, which provides a platform for sharing knowledge and ideas, can be imperative for innovation.

KWs are interdependent, which makes it difficult to contribute specific value to specific organizational members. Value is often created by helping others, sharing knowledge, and delivering results in a timely manner so that others can use them to generate value. Helping others not only has the potential to contribute to value creation for the organization, but builds relationships and trust as well. Relationships can be a source of well-being and give the KW access to information and knowledge.

It is important that KWs share knowledge to contribute to the learning systems of the organization, which are the basis of the organizational knowledge base. The organizational knowledge base can give the organization a competitive advantage. Organizations need to have a climate of supportiveness, belongingness, and mutual trust for their KW's to be able to create a shared cognitive frame, which is necessary for knowledge transfer. It is beneficial to the organization to encourage knowledge sharing and helping behaviors. 
Table 2 shows an overview of the key takeaways of this paper that have implications for managing and improving KWP.

There is a need for further research before applicable frameworks and methods can be found to manage, measure, and improve KWP holistically. It is apparent, from the interdependence of KWs, the subjectivity of value and effort, as well as the latency of some value, that measuring KWP is a challenge. It is difficult to know if initiatives or methods used to manage KWP are successful if KWP cannot be measured effectively. Therefore, there is a need to define and develop ways to measure KWP that consider this subjectivity and latency.

It would be interesting in future research to fully explore the how of the system and create an applicable framework that can be tested. Mapping popular management strategies to the purposeful activity model, to analyze what activities they are targeting and how, would give insight into how they could be combined to create a holistic strategy to manage and improve KWP.

Table 2. Key takeaways.

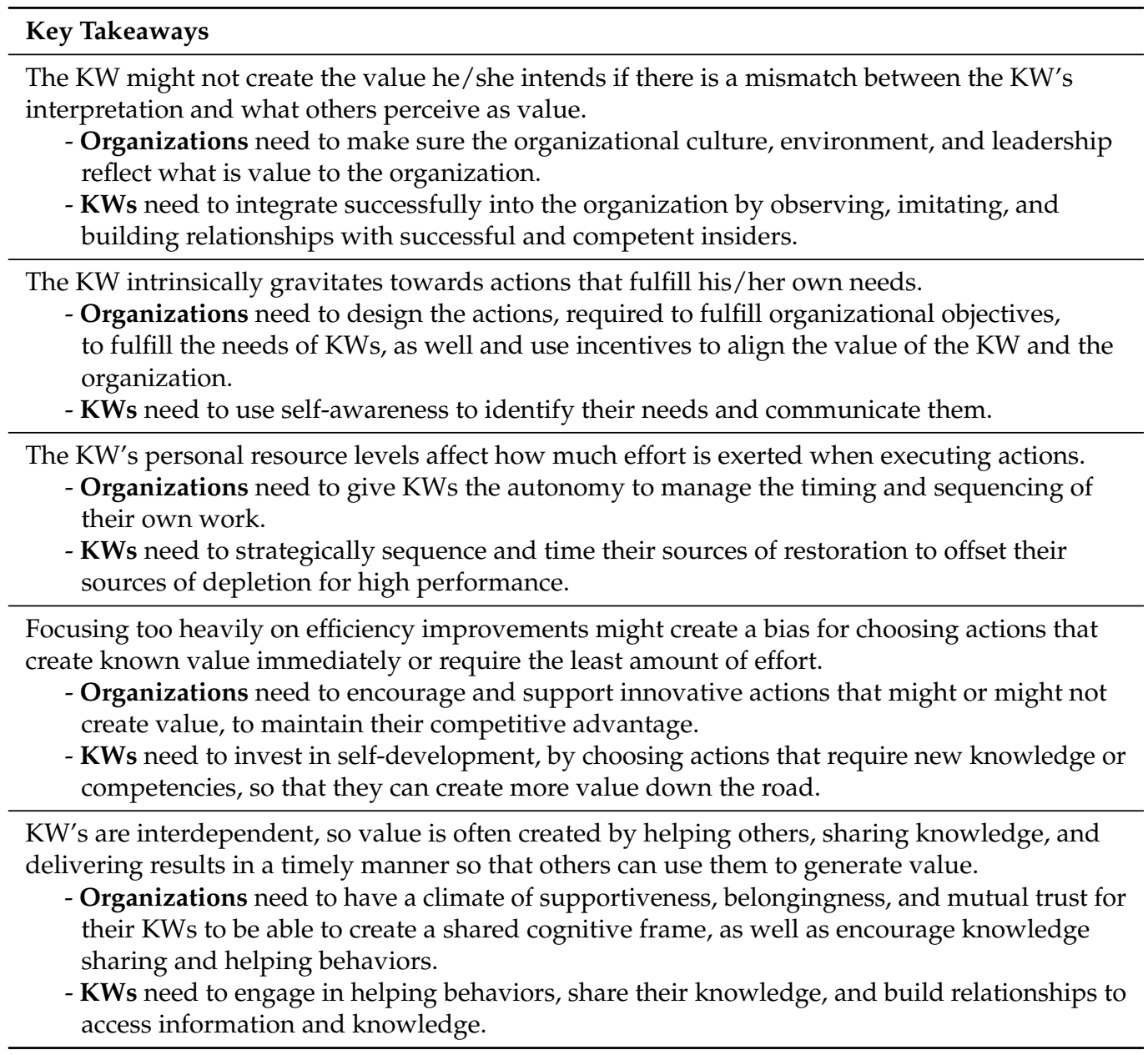

Another idea for future research is to map different KW behaviors to the purposeful activity model and analyze what activities are omitted and why. For example, a common behavior pattern of a KW is that of withholding knowledge to make themselves indispensable. Those who partake in that behavior are not executing the activities communicate results of actions to relevant parties or share knowledge acquired while executing actions. These individuals might seem very productive, that is they probably generate many artifacts. However, value is not created unless these artifacts are delivered to someone who then utilizes them. These kinds of behaviors are often the result of the cultural environment in 
the organization where, for example, competition between workers is intensified through badly designed bonus programs. This is an example of how the KW is confined and influenced by the systems in his/her environment. It stresses the importance of looking at both problem owners, the individual and the organization, when exploring how to manage and improve KWP.

According to the path set by Óskarsdóttir and Oddsson (2017), the next step in this research is to formulate a purposeful activity model of the system owned by the organization. Only after both of these viewpoints have been explored can the situation be debated and actions for improvement identified. These are the last two activities in the SSM. Hopefully, applicable frameworks and methods can then be created from the identified actions for improvement and knowledge gained from this research.

The vastness of current research and the level of detail, which touch on factors that influence KWP, had a limiting effect on this research, making it difficult to obtain a complete holistic view. The results of the first paper, which analyzed the problem situation, were used to limit the scope. The purposeful activity model was formulated from interpretations and inferences made from the literature review.

Managing and improving KWP are complicated by the fact that knowledge resides in the minds of KWs and cannot easily be assimilated into the organization's process. Any approach, framework, or method to manage and improve KWP needs to give consideration to the human nature of KWs, which influences their productivity. This paper highlighted the individual KW's role in managing and improving KWP by exploring the process in which he/she creates value.

Author Contributions: H.G.Ó. and G.V.O. conceived of and designed the research; H.G.Ó. performed the research, created the model, and wrote the paper. J.P.S. and R.J.S. reviewed the paper. All authors have read and agreed to the published version of the manuscript.

Funding: This research received no external funding.

Institutional Review Board Statement: Not applicable.

Informed Consent Statement: Not applicable.

Data Availability Statement: Not applicable.

Conflicts of Interest: The authors declare no conflict of interest.

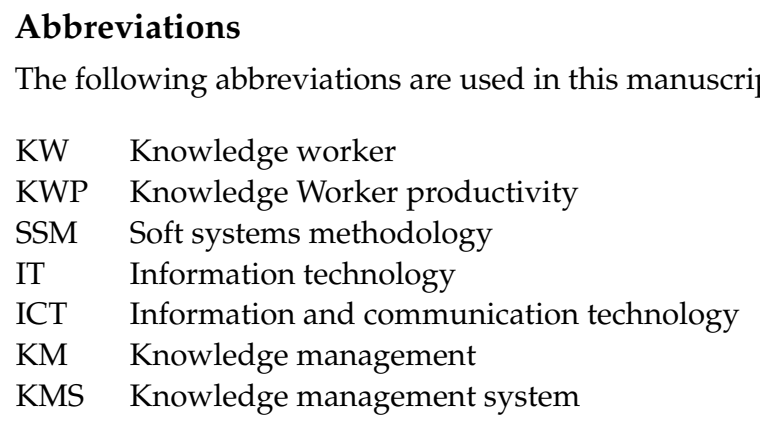

\section{References}

Aboagye, Emmanuel, Christina Björklund, Klas Gustafsson, Jan Hagberg, Gunnar Aronsson, Staffan Marklund, Constanze Leineweber, and Gunnar Bergström. 2019. Exhaustion and impaired work performance in the workplace: Associations with presenteeism and absenteeism. Journal of Occupational and Environmental Medicine 61: e438-44. [CrossRef] [PubMed]

Alimo-Metcalfe, Beverly, John Alban-Metcalfe, Margaret Bradley, Jeevi Mariathasan, and Chiara Samele. 2008. The impact of engaging leadership on performance, attitudes to work and wellbeing at work: A longitudinal study. Journal of Health Organization and Management 22: 586-98. [CrossRef] [PubMed]

Amar, Amar D. 2004. Motivating knowledge workers to innovate: A model integrating motivation dynamics and antecedents. European Journal of Innovation Management 7: 89-101. [CrossRef]

Bogdanowicz, Maureen S., and Elaine K. Bailey. 2002. The value of knowledge and the values of the new knowledge worker: Generation $x$ in the new economy. Journal of European Industrial Training 26: 125-29. [CrossRef] 
Borman, Walter C., and Stephan J. Motowidlo. 1997. Task performance and contextual performance: The meaning for personnel selection research. Human Performance 10: 99-109. [CrossRef]

Bowman, Cliff, and Véronique Ambrosini. 2000. Value creation versus value capture: Towards a coherent definition of value in strategy. British Journal of Management 11: 1-15. [CrossRef]

Boyatzis, Richard. 2008. Competencies in the 21st century. Journal of Management Development 27. [CrossRef]

Boyatzis, Richard E. 1982. The Competent Manager. New York: John Wiley and Sons, Inc.

Cake, Martin A., Michelle M. McArthur, Susan M. Matthew, and Caroline F. Mansfield. 2017. Finding the balance: Uncovering resilience in the veterinary literature. Journal of Veterinary Medical Education 44: 95-105. [CrossRef]

Checkland, Peter. 1993. Systems Thinking, Systems Practice. Chichester: John Wiley and Sons Ltd.

Checkland, Peter. 2000. Soft systems methodology: A thirty year retrospective. Systems Research and Behavioral Science 17: 11-58. [CrossRef]

Checkland, Peter. 2011. Autobiographical retrospectives: Learning your way to 'action to improve' - The development of soft systems thinking and soft systems methodology. International Journal of General Systems 40: 487-512. [CrossRef]

Cooper-Thomas, Helena, and Neil Anderson. 2006. Organizational socialization: A new theoretical model and recommendations for future research and hrm practices in organizations. Journal of Managerial Psychology 21: 492-516. [CrossRef]

Cropanzano, Russell, Deborah Rupp, and Zinta Byrne. 2003. The relationship of emotional exhaustion to job performance ratings and organizational citizenship behavior. The Journal of Applied Psychology 88: 160-69. [CrossRef] [PubMed]

Drucker, Peter F. 1999. Knowledge-worker productivity: The biggest challenge. California Management Review 41: 79-94. [CrossRef]

Drucker, Peter F., Esther Dyson, Charles Handy, Paul Saffo, and Peter M. Senge. 1997. Looking ahead: Implications of the present. Harvard Business Review 75: 18-27. [PubMed]

Ebert, Philip, and Wolfgang Freibichler. 2017. Nudge management: Applying behavioural science to increase knowledge worker productivity. Journal of Organization Design 6: 1-6. [CrossRef]

Edmunds, Angela, and Anne Morris. 2000. The problem of information overload in business organisations: A review of the literature. International Journal of Information Management 20: 17-28. [CrossRef]

Eriksson, Andrea, Arne Orvik, Margaretha Strandmark, Anita Nordsteien, and Steffen Torp. 2017. Management and leadership approaches to health promotion and sustainable workplaces: A scoping review. Societies 7: 14. [CrossRef]

Fiol, C. Marlene, and Marjorie A. Lyles. 1985. Organizational learning. The Academy of Management Review 10: 803-13. [CrossRef]

Fritz, Charlotte, Chak Fu Lam, and Gretchen Spreitzer. 2011. It's the little things that matter: An examination of knowledge workers' energy management. Academy of Management Perspectives 25: 28-39. [CrossRef]

Garavan, Thomas, and David Mcguire. 2001. Competencies and workplace learning: Some reflections on the rhetoric and the reality. Journal of Workplace Learning 13: 144-64. [CrossRef]

Goleman, Daniel. 1999. Working with Emotional Intelligence. London: Bloomsbury Publishing Plc.

Gonzalez, Rodrigo, and Manoel Martins. 2014. Knowledge management: An analysis from the organizational development. Journal of Technology Management $\mathcal{E}$ Innovation 9: 131-47. [CrossRef]

Greenblatt, Edy. 2002. Work/life balance: Wisdom or whining. Organizational Dynamics 31: 177-93. [CrossRef]

Hall, Douglas T. 2004. Self-Awareness, Identity, and Leader Development. Series in Applied Psychology; Mahwah: Lawrence Erlbaum Associates Publishers, pp. 153-76.

Hemp, Paul. 2009. Death by information overload. Harvard Business Review 87: 82-89. [PubMed]

Hobfoll, Stevan. 1989. Conservation of resources: A new attempt at conceptualizing stress. The American Psychologist 44: 513-24. [CrossRef] [PubMed]

Hobfoll, Stevan, Robert Johnson, Nicole Ennis, and Anita Jackson. 2003. Resource loss, resource gain, and emotional outcomes among inner city women. Journal of Personality and Social Psychology 84: 632-43. [CrossRef] [PubMed]

Jia, Huiyuan, Rui Zhong, and Xiaofei Xie. 2020. Helping others makes me fit better: Effects of helping behavior by newcomers and coworker-attributed motives on newcomers' adjustment. Journal of Business and Psychology 36: 401-16. [CrossRef]

Kang, Sung-Choon, Shad S. Morris, and Scott A. Snell. 2007. Relational archetypes, organizational learning, and value creation: Extending the human resource architecture. Academy of Management Review 32: 236-56. [CrossRef]

Kira, Mari M., Frans van Eijnatten, and David B. Balkin. 2010. Crafting sustainable work: Development of personal resources. Journal of Organizational Change Management 23: 616-32. [CrossRef]

Kline, Stephen J., and Nathan Rosenberg. 2009. An overview of innovation. In Studies on Science and the Innovation Process: Selected Works of Nathan Rosenberg; pp. 173-203. [CrossRef]

Kroft, Steven H. 2020. Well-Being, Burnout, and the Clinical Laboratory. American Journal of Clinical Pathology 153: 422-24. [CrossRef]

Kundapur, Poornima, and Lewlyn L. R. Rodrigues. 2012. Simulating knowledge worker adoption rate of kms: An organizational perspective. International Journal of Innovation, Management and Technology 3: 459. [CrossRef]

Lee, Ching, and Jie Yang. 2000. Knowledge value chain. Journal of Management Development 19: 783-94. [CrossRef]

Letiche, Hugo, and René van Hattem. 2000. Self and organization-Knowledge work and fragmentation. Journal of Organizational Change Management 13: 352-74. [CrossRef]

Livi, Stefano, Annalisa Theodorou, Marika Rullo, Luigi Cinque, and Guido Alessandri. 2018. The rocky road to prosocial behavior at work: The role of positivity and organizational socialization in preventing interpersonal strain. PLoS ONE 13: e193508. [CrossRef]

Loehr, Jim, and Tony Schwartz. 2001. The making of a corporate athlete. Harvard Business Review 79: 120-28. 
Louis, Meryl Reis. 1980. Surprise and sense making: What newcomers experience in entering unfamiliar organizational settings. Administrative Science Quarterly 25: 226-51. [CrossRef] [PubMed]

Maier, Ronald. 2007. Knowledge Management Systems, 3rd ed.; Berlin: Springer.

Maslach, Christina, and Michael P. Leiter. 1997. The Truth about Burnout: How Organizations Cause Personal Stress and What to Do about It. San Francisco: Jossey-Bass.

Michael, James, and Gary Yukl. 1993. Managerial level and subunit function as determinants of networking behavior in organizations. Group E Organization Management 18: 328.

Mládková, Ludmila. 2011. Knowledge management for knowledge workers: Ejkm. Electronic Journal of Knowledge Management 9: 248-58.

Nonaka, Ikujiro. 1994. A dynamic theory of organizational knowledge creation. Organization Science 5: 14-37. [CrossRef]

Nonaka, Ikujiro, and Toyama Ryoko. 2003. The knowledge-creating theory revisited: Knowledge creation as a synthesizing process. Knowledge Management Research \& Practice 1: 2.

Óskarsdóttir, Helga Guðrún, and Guðmundur Valur Oddsson. 2017. A soft systems approach to knowledge worker productivity-Analysis of the problem situation. Economies 5: 28. [CrossRef]

Oyefolahan, Ishaq, and Panneer Dhanapal Durai Dominic. 2013. Knowledge management systems use and knowledge workers competency development: The role of socio-technical antecedents in developing autonomous motivation to use. Journal of Information \& Knowledge Management 43: 482-500. [CrossRef]

Peseschkian, Hamid, and Arno Remmers. 2020. Life balance with positive psychotherapy. In Positive Psychiatry, Psychotherapy and Psychology. Edited by Hamid Peseschkian and Consuelo Cagande. Cham: Springer, vol. 8, pp. 91-9.

Podsakoff, Philip M., Michael Ahearne, and Scott B. MacKenzie. 1997. Organizational citizenship behavior and the quantity and quality of work group performance. Journal of Applied Psychology 82: 262-70. [CrossRef] [PubMed]

Polanyi, Michael. 1966. The Tacit Dimension. Garden City: Doubleday.

Popper, Micha, and Raanan Lipshitz. 1998. Organizational learning mechanisms: A structural and cultural approach to organizational learning. The Journal of Applied Behavioral Science 34: 161-79. [CrossRef]

Rittel, Horst W. J., and Melvin M. Webber. 1973. Dilemmas in a general theory of planning. Policy Sciences 4: 155-69. [CrossRef]

Robertson, Ivan, and Jill Flint-Taylor. 2009. Leadership, Psychological Well-Being, and Organizational Outcomes. Oxford: Oxford University Press. [CrossRef]

Rosendaal, Bastiaan. 2009. Sharing knowledge, being different and working as a team. Knowledge Management Research E Practice 7: $4-14$.

Ryan, Geoff, Robert J. Emmerling, and Lyle M. Spencer. 2009. Distinguishing high-performing european executives: The role of emotional, social and cognitive competencies. The Journal of Management Development 28: 859-75. [CrossRef]

Salvagioni, Denise Albieri Jodas, Francine Nesello Melanda, Arthur Eumann Mesas, Alberto Durán González, Flávia Lopes Gabani, and Selma Maffei de Andrade. 2017. Physical, psychological and occupational consequences of job burnout: A systematic review of prospective studies. PLoS ONE 12: e0185781. [CrossRef]

Seethamraju, Ravi. 2000. Knowledge management issues in the management of professional engineers. Journal of Scientific $\mathcal{E}$ Industrial Research 59: 710-19.

Shalini, Grace, Abdul Basit, and Zubair Hassan. 2018. The impact of social media usage on employee and organization performance: A study on social media tools used by an it multinational in malaysia. Journal of Marketing and Consumer Behaviour in Emerging Markets 1: 48-65. [CrossRef]

Smith, C. Ann, Dennis W. Organ, and Janet P. Near. 1983. Organizational citizenship behavior: Its nature and antecedents. Journal of Applied Psychology 68: 653-63. [CrossRef]

Spencer, Lyle M., and Phd Signe M. Spencer. 1993. Competence at Work: Models for Superior Performance. New York: John Wiley and Sons, Inc.

Urbancova, Hana, Lucie Vnouckova, and Sarka Laboutkova. 2016. Knowledge transfer in a knowledge-based economy. E E M Ekonomie A Management 19: 73-86. [CrossRef]

Van der Vegt, Gerben, and Evert Van de Vliert. 2002. Intragroup interdependence and effectiveness: Review and proposed directions for theory and practice. Journal of Managerial Psychology 17: 50-67. [CrossRef]

Van Scotter, James R., and Stephan J. Motowidlo. 1996. Interpersonal facilitation and job dedication as separate facets of contextual performance. Journal of Applied Psychology 81: 525-31. [CrossRef]

Van Zyl, Anria. 2009. The impact of social networking 2.0 on organisations. The Electronic Library 27: 906-18. [CrossRef]

Weiss, Howard M. 1978. Social learning of work values in organizations. Journal of Applied Psychology 63: 711-18. [CrossRef]

Zwikael, Ofer, and John Smyrk. 2012. A general framework for gauging the performance of initiatives to enhance organizational value. British Journal of Management 23: S6-S22. [CrossRef] 